\title{
A GENERIC WAKE ANALYSIS TOOL AND ITS APPLICATION TO THE JAPAN BULK CARRIER TEST CASE
}

\section{Matthias Maasch, Kurt Mizzi, Mehmet Atlar, Patrick Fitzsimmons, Osman Turan}

Department of Naval Architecture and Marine Engineering, University of Strathclyde, 100 Montrose Street, Glasgow G4 OLZ, UK, matthias.maasch@strath.ac.uk, kurt.mizzi@strath.ac.uk, Mehmet.atlar@strath.ac.uk, patrick.a.fitzsimmons@gmail.com, o.turan@strath.ac.uk

\begin{abstract}
Economic pressures and regulatory obligations have brought about a great interest in improving ship propulsion efficiency. This has motivated and directed research to improve the hydrodynamic performance of a vessel thus seeking the development and generation of optimised designs. When analysing flow characteristics of a vessel, particular attention is given to analysing its wake field and stern flow characteristics. For the detailed analysis of the wake flow field, a Wake Analysis Tool (WAT) was developed within the programming environment of a commercial CAE tool. The WAT is based on a framework that enables easy interaction to an external CFD solver reading flow field properties from a generic csv file. The wake flow field is visualised and analysed in schematic form according to the standard and customized (i.e. the British Ship Research Association criteria) wake analyses. By coupling the CAE tool with a commercial flow solver, an example was given how to use the wake tool in a fully automated simulation process. Finally, the wake tool was used to analyse the nominal propeller wake flow of the Japan Bulk Carrier (JBC) thus showing how it can be efficiently applied to a set of numerical results.
\end{abstract}

Keywords: CFD, Wake Analysis Tool, Ship Design Automation, RANSE, Japan Bulk Carrier 


\section{Wake Theory}

$c_{B} \quad$ Ship block coefficient

$D \quad$ Propeller diameter

$d w / \mathrm{d} \varphi \quad$ Wake gradient

$g \quad$ Gravitational acceleration

$H_{i} \quad$ Propeller tip clearance

$N \quad$ Propeller rotation rate

$P_{a} \quad$ Atmospheric pressure

$P_{I} \quad$ Hydrostatic pressure

$P_{v} \quad$ Saturated vapour pressure of

water

$r \quad$ Local propeller radius

$R \quad$ Propeller radius

$r / R \quad$ Dimensionless radius

$T_{A} \quad$ Ship draft

$v \quad$ Ship speed

$v_{a}(r, \varphi) \quad$ Axial velocity

$v_{r}(r, \varphi) \quad$ Radial velocity

$v_{t}(r, \varphi) \quad$ Tangential velocity

$v_{w}(r, \varphi) \quad$ Wake Velocity

$w \quad$ Wake

$w_{\Delta} \quad$ Wake non-uniformity

$\bar{w} \quad$ Local average wake

$Z_{p} \quad$ Distance between propeller shaft

axis and base line

$\Delta w \quad$ Wake variation

$\rho \quad$ Water density

$\sigma_{n I} \quad$ Tip cavitation number based on

$\sigma_{n I} \quad$ shaft speed

$\varphi \quad$ Wake angle

Ship Particulars

$B W L \quad$ Beam at waterline

$c_{B} \quad$ Block coefficient

$D \quad$ Depth

$L P P \quad$ Length between perpendiculars

$L W L \quad$ Length at waterline

$S_{\text {Baseline }} \quad$ Wetted surface of JBC without duct

$S_{E S D} \quad$ Wetted surface of JBC hull with

duct

$T_{A} \quad$ Ship draft

$\nabla \quad$ Displacement

$\Lambda \quad$ Model scale factor

\section{Navier Stokes Equations}

$\bar{p} \quad$ Mean pressure

$t \quad$ Time

$\bar{u}_{i} \quad$ Averaged Cartesian velocity

vector component $(i)$

$\bar{u}_{j} \quad$ Averaged Cartesian velocity

$\bar{u}_{j} \quad$ vector component $(j)$

$x_{i} \quad$ Cartesian coordinate $(i)$

$x_{j} \quad$ Cartesian coordinate $(j)$

$\mu \quad$ Dynamic viscosity

$\bar{\tau}_{i j} \quad$ Mean viscous stress tensor

$\rho \overline{u_{i} \hat{u}_{j}} \quad$ Reynolds stress

\section{Validation and Verification}

CF Correction factor

CGI Grid convergence index

$D / E F D \quad$ Experimental output

E Comparison error

$p \quad$ Order of accuracy

$p_{\text {est }} \quad$ Estimated order of accuracy

$r_{G} \quad$ Parameter refinement ratio

$R_{G} \quad$ Convergence ratio

$S_{1,2,3} \quad$ Solver output

$T \quad$ True value

$U_{D} \quad$ Experimental uncertainty

$U_{G} \quad$ Grid uncertainty

$U_{S M} \quad$ Modelling uncertainty

$U_{S N} \quad$ Numerical uncertainty

$U_{v} \quad$ Validation uncertainty

$\delta_{D} \quad$ Experimental error

$\delta_{G} \quad$ Grid error

$\delta_{S} \quad$ Simulation error

$\delta_{S M} \quad$ Modelling error

$\delta_{S N} \quad$ Numerical error 


\section{Introduction}

CFD codes have advanced and been developed significantly in the past years opening doors to novel ship performance prediction methods. The capability of simulating a complex ship hull that is free to move in a virtual towing tank environment has exploited new areas of research including the assessment of flow details that were impossible to investigate due to solver and power limitations. Different studies focus on different areas of investigation, with some analysing performance parameters such as ship hull resistance, propulsive power and motions as well as others seeking detailed wake flow characteristics and behaviour.

Assessing the quality of the wake flow is a pivotal process in the design procedure. The stern flow characteristics and fluid flow behaviour have an impact on both the hull and propeller systems. The hull efficiency is dependent on the design of the vessel that dictates the inflow to the propeller and hence the propulsive efficiency. In turn, the hull-propeller interaction also gives rise to pressure excited vibrations (PEV) that have to be kept to acceptable levels for safety purposes. A ship advancing through water causes a non-uniform flow field, i.e. wake field, behind it. The disturbed velocity distribution of the wake field at the propeller plane is influenced by the streamline flow around the ship hull in front of the propeller, the extent of the boundary layer caused by the fluid viscosity and the effect of the disturbed free surface. A detailed measurement of the propeller wake field is necessary to custom-design marine propellers. However, it is estimated that around $80-85 \%$ of the new-build ships do not undergo an experimental model wake field test. In order to experimentally measure the wake field velocities, i.e. Experimental Fluid Dynamics (EFD), two methods are typically used by model basins/ towing tank facilities. An intrusive method is to place a five-hole Pitot tube rake onto the shaft in the propeller plane (see Figure 1). [1]
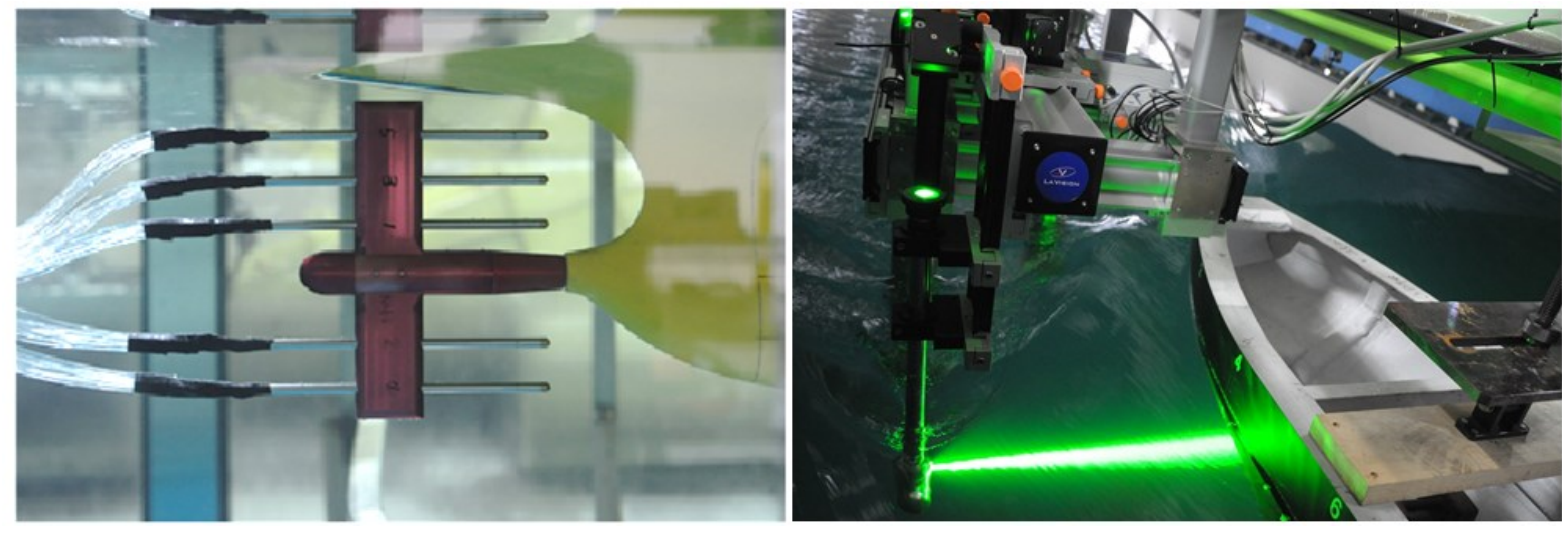

Figure 1 - Devices for experimentally measuring the nominal wake field in the propeller plane, (LHS) Pitot rake, (RHS) PIV - Particle Image Velocimetry [2]

The Pitot tubes measure the flow pressure distribution which can be used to derive the flow velocities by applying Bernoulli's equation. In recent years Laser Doppler Velocimetry (LDA) (e.g. [3]) and Particle Image Velocimetry (PIV) techniques (e.g. [4]) improved the quality of the measurements as these nonintrusive methods do not disturb the fluid flow by the presence of the device. [1]

Once the velocity distribution in the propeller plane is measured, methods can be applied to calculate wake field quality criteria by using representative wake parameters such as the wake fraction. This gives insight into the performance prediction of the propulsor and also allows predicting the propellerhull interaction phenomena which affects the propulsive efficiency and pressure pulses on the hull. In order to relate the analysis to propeller design theory, measurement points are often placed in polar coordinates, so that the measurement outcome can be expressed as radial velocity distributions. This allows predicting the velocity vector that a propeller blade section is facing during rotation. A typical polar coordinate convention of such a wake disc is shown in Figure 2. 


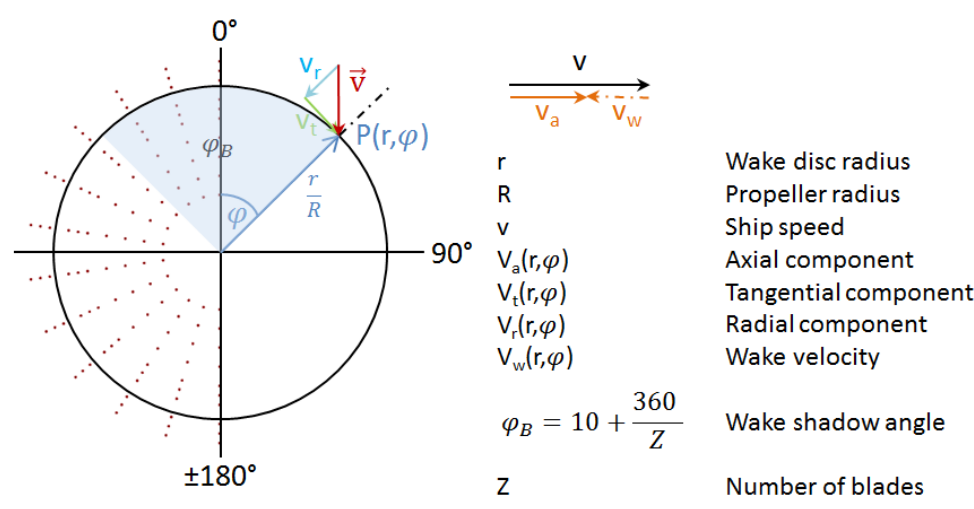

Figure 2 - Wake field velocities and exemplary measure points (red) of propeller wake field

The above figure defines the wake disc using a polar/cylindrical coordinate system. The disc coordinate $P$ is defined by the disc radius $r$ and the wake angle $\varphi$ starting from the top dead centre position running clockwise (looking into the propeller plane from behind). The velocity vector $\vec{v}$ is composed of its axial component $v_{a}$ facing normal to the propeller plane, its tangential component $v_{t}$ and its radial component $v_{r}$. The wake velocity $v_{w}$ is the difference of ship speed and the axial velocity component. The radius of the wake disc equals the radius of the propeller, expressed as dimensionless radius $\frac{r}{R}$ with $R$ being the propeller radius, whereas the exemplary measure points indicate that the wake field measurement should exceed the propeller radius. Special interest lies in the top dead centre and its proximity which is known to be the wake shadow area and its extend is defined by wake shadow angle $\varphi_{B}$. Computational Fluid Dynamics (CFD) allows us to numerically estimate the flow pressure and velocity distribution in the ship wake field enabling analysis to be carried out in a similar way to the experimental approach. A major advantage of CFD is that the flow field can be measured in a very detailed manner up to the resolution of the numerical mesh. Furthermore, studies can be carried out in nominal (i.e. no propeller action is included) as well as in self-propulsion conditions.

Carlton [5] indicated that wake irregularity (non-uniformity) has a significant influence on the ship aft end vibrations. A particular wake field area of interest that is susceptible to cavitation is the wake peak inside the wake shadow (see blue shaded area in Figure 2) where the propeller is subject to heavy loads. Furthermore, once the hull form has been established, wake flow analyses are used to determine the design of the propeller. All these factors are directly related to the energy efficiency of the vessel making wake quality assessment a crucial process. Wake quality analysis procedures can be generally divided into two approaches; analytical methods and heuristic methods. The latter, which are the more popular methods of the two, make use of the axial velocity components whereas the analytical methods utilise extensive wake field data to investigate the quality in all three measured directions. Regarding Heuristic approaches, Huse [6] developed a set of criteria based on axial fluid flow characteristics in the absence of the propeller excluding any issues associated with pressures and vibrations. Odabasi and Fitzsimmons [7] have therefore extended Huse's work to consider vibrations and hull surface pressures by levels of vibration measured on a set of towed ship models. Although the discussed methods are based on empirical formulae, these criteria can be considered as useful guidelines and indicators for a preliminary investigation of hull-propeller interaction.

In recent years, numerical procedures have become capable of predicting details of ship stern flow behaviour. Larsson [8] explain that the type of turbulence model plays a crucial role in wake prediction. Visonneau [9] and Hanninen [10] continue to add that it's the turbulence closure that dictates the level of detail in wake flow prediction. Outcomes from the Gothenburg 2010 workshop demonstrate the significant progress in predicting flow characteristics aft of U-shaped hulls and that most codes produced reasonably accurate results when compared with experimental data. They too support the argument that turbulence models play an important role but continue to add that the grid resolution 
should be sufficiently fine to capture certain details. Wang [11] indicated that the Reynolds Stress Model (RSM) is the best turbulence model to capture detailed wake flow characteristics such as bilge vortex. The EFFORT project [9] was also carried out to compare the suitability of different turbulence models and highlighted certain outcomes as follows. The $k-\omega$ and $k-\varepsilon$ two equation turbulence models successfully managed to capture the general flow around the ship. The models however demonstrated their inability to predict certain wake flow characteristics, especially vortex structures. The more sophisticated RSM models are better suited to capturing and simulating stronger bilge vortices but are however computationally expensive and less robust than the SST k- $\omega / \mathrm{k}-\varepsilon$ models. For a larger set of simulations the SST two equation turbulence models were deemed more adequate for solving wake characteristics providing a good compromise between $k-\omega / k-\varepsilon$ methods and more complex RSM models. Optimising hull forms and propeller designs are not the only strategies to reduce propeller induced vibrations and improve propulsion efficiency. The installation of Energy Saving Devices (ESD) can also contribute to the cause by improving the inflow to the propeller. The wide range of Energy Saving Devices can be classified under different categories and different working principles [12]. For their optimal function, one should understand and analyse the impact of the ESD on the wake flow of the vessel thus making wake quality assessment procedures also crucial for the installation of retrofitting technologies. Studies $[13,14]$ analysing the impact of Wake Equalizing Ducts (WEDs) indicate the acceleration of the water in the wake peak area producing a more uniform flow and reduced maximum wake fraction. Furthermore, others have also investigated the function of vortex generator fins $[15,16]$.

This present work introduces the functions and the proposed workflow of the developed Wake Analysis Tool (WAT) along with its application by performing a wake field analysis study on the stern flow of the Japan Bulk Carrier in nominal resistance conditions. The tool was already briefly presented in [17] showing its capacities by assessing nominal wake fields of an LNG Carrier in level and extreme trim conditions. First, the development framework of the WAT is presented, highlighting its universal use. Second, the implemented basic wake quality criteria are presented followed by a customised extension of the code. Third, the Japan Bulk Carrier (JBC) [18] case study is introduced along with a validation and verification study of the numerical towing tank setup following the method of Xing and Stern [19]. Fourth, a wake analysis pre-processing tool is presented which allowed automating the wake field analysis using FRIENDSHIP System's CAE tool CAESES and SIEMENS' flow solver STAR-CCM+. Fifth, results of the JBC stern flow are briefly analysed followed by a comprehensive nominal wake field analysis using the developed wake analysis tool. The case study process is visualised in Figure 3. Finally, a short conclusion evaluates the use of the wake tool and points to additional fields of application and future use.

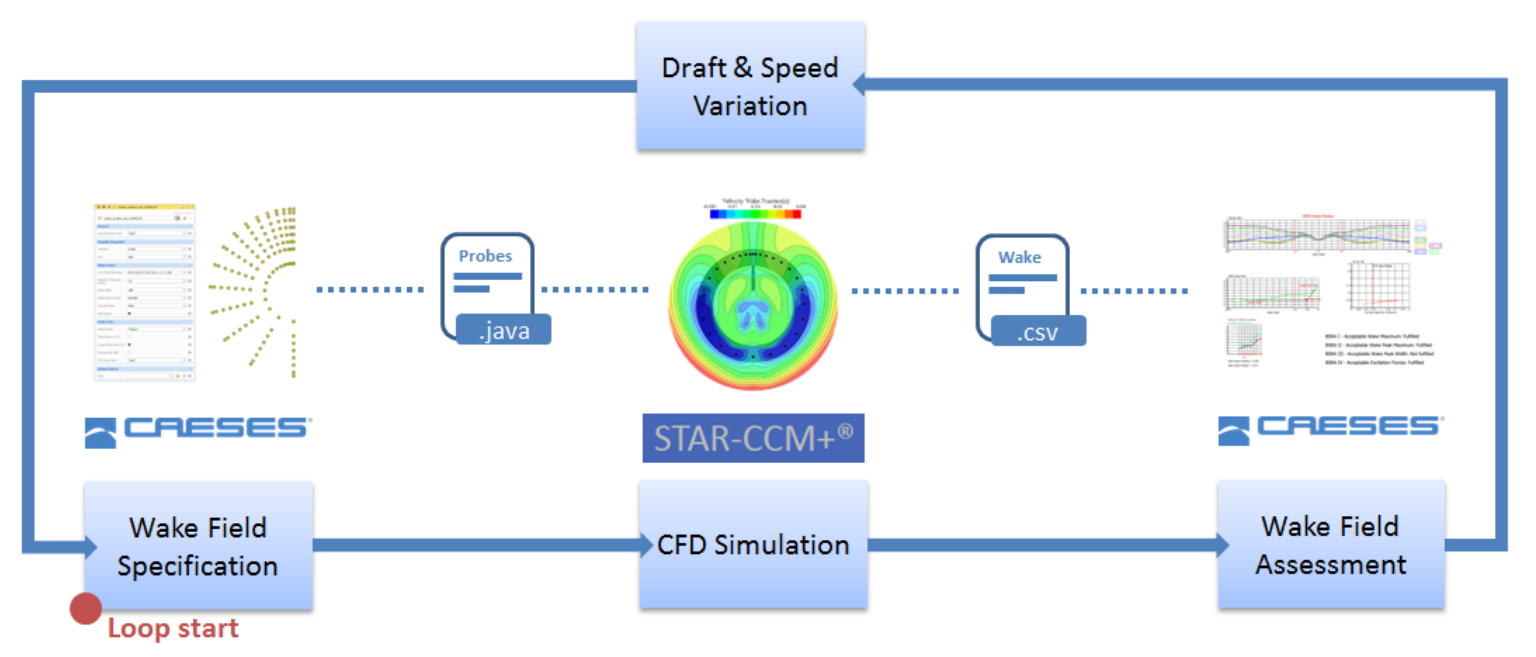

Figure 3 - Case Study Process Scheme 


\section{WAT - Wake Analysis Tool}

The Wake Analysis Tool (WAT) was developed within the programming environment of the CAE tool that allows generating custom codes by giving access to the entire software command set. This includes file import and export, the visualisation of curves in a 3D window as well as the numerical analysis of the given input.

A comma-separated flow field data file (CSV) provided by an external flow solver gets imported and read by the WAT. The presented code accepts various data structures within the given CSV file as the file-reading process can be customised. The visualisation output and the type of analysis can be controlled via the WAT Graphical User Interface (GUI). The results of the numerical analysis are accessible via output parameters that can further be used to vary and optimise the underlying geometry or operational conditions. Finally, the results can be exported as a CSV file and also be displayed in graphical form. As the code is accessible, custom data analyses can be integrated as an extension, e.g. the later presented B.S.R.A. (British Ship Research Association) wake quality criteria.

For most CFD flow solvers the generation of a CSV file containing particular flow data (e.g. velocities on a plane within the flow domain) can be automated. However, the structure of the given data differs from code to code due to the included headings and additional data such as grid coordinates. To provide an analysis tool that suits various CSV file formats, the reading process of the presented WAT is kept generic and can be customized.

\section{BASIC WAKE QUALITY ASSESSMENT}

The assessment of the wake field follows a commonly used heuristic method that takes the given velocity ratios $\frac{v_{i}}{v}\left(v_{i}=v_{a}, v_{t}, v_{r}\right)$ independently into account. From the given input file the WAT plots the velocity ratio $\frac{v_{i}}{v}$ over the given wake angle range $\Delta \varphi$. The WAT also accepts input files that only provide half of a wake field (this can be a result of a nominal half-domain towing tank simulation). The given velocity ratio distributions are analysed deriving the maximum velocity variation (1) and the averaged velocity ratio (2) for each given radius.

$$
\begin{gathered}
\Delta\left(\frac{v_{i}}{v}\right)_{r}=\left(\frac{v_{i}}{v}\right)_{r, \max }-\left(\frac{v_{i}}{v}\right)_{r, \min } \\
\overline{\left(\frac{v_{l}}{v}\right)_{r}}=\frac{1}{\Delta \varphi} \int_{\varphi_{\min }}^{\varphi_{\max }}\left(\frac{v_{i}}{v}\right)_{r} d \varphi
\end{gathered}
$$

The mean wake variation (3) and the mean wake fraction (4) on the wake disc are calculated and made accessible as visual and numerical outputs which can be used as evaluation parameters in a variation and optimisation study. The mean wake variation (3) takes the variation of the inflow on each radius equally into account.

$$
\Delta w=\frac{1}{\left(r_{\max }-r_{\min }\right)} \int_{r_{\min }}^{r_{\max }} \Delta\left(1-\frac{v_{i}}{v}\right)_{r} d r
$$

The mean Taylor wake fraction (4) is integrated on a volumetric basis over the given wake disc.

$$
w=\frac{1}{\Delta \varphi\left(r_{\max }^{2}-r_{\min }^{2}\right)} \int_{r_{\min }}^{r_{\max }} r \int_{\varphi_{\min }}^{\varphi_{\max }}\left(1-\frac{v_{i}}{v}\right)_{r} d \varphi d r
$$


As already mentioned above, the analysis can be performed for each velocity component, depending on which row/column is selected in the GUI. The final output of the WAT (Figure 4) is composed of a (\#1) graphical output, (\#2) a detailed console output and (\#3) a text file that holds general information such as project name, date, number of radii and number of points as well as each calculated value in table format.

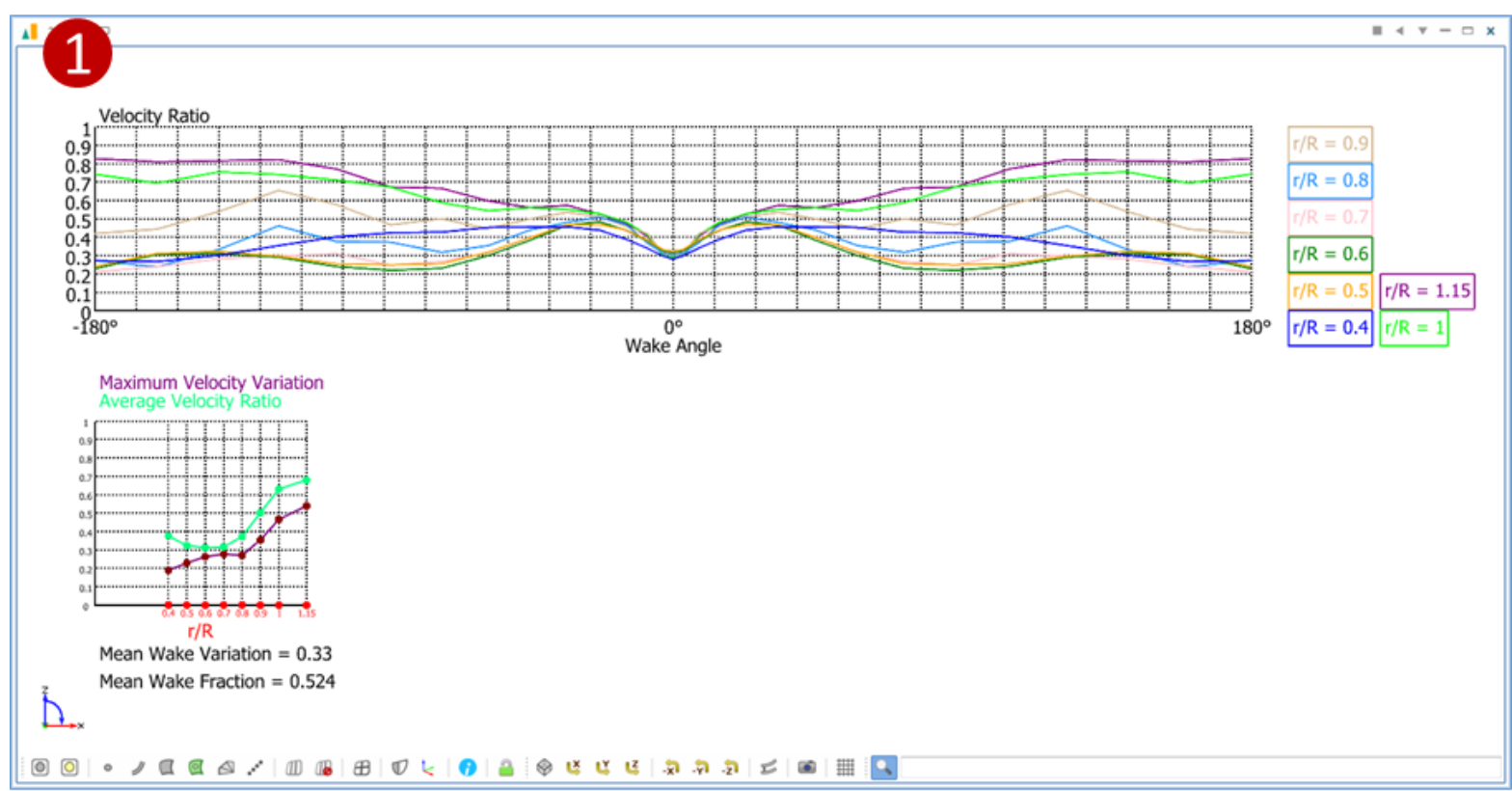

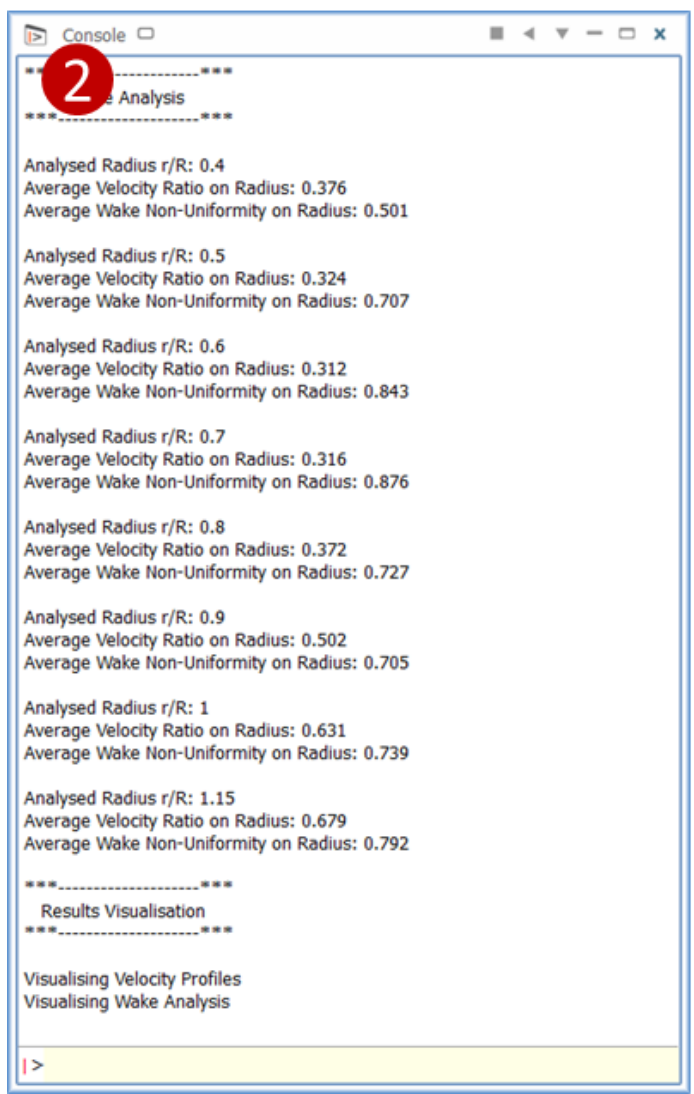

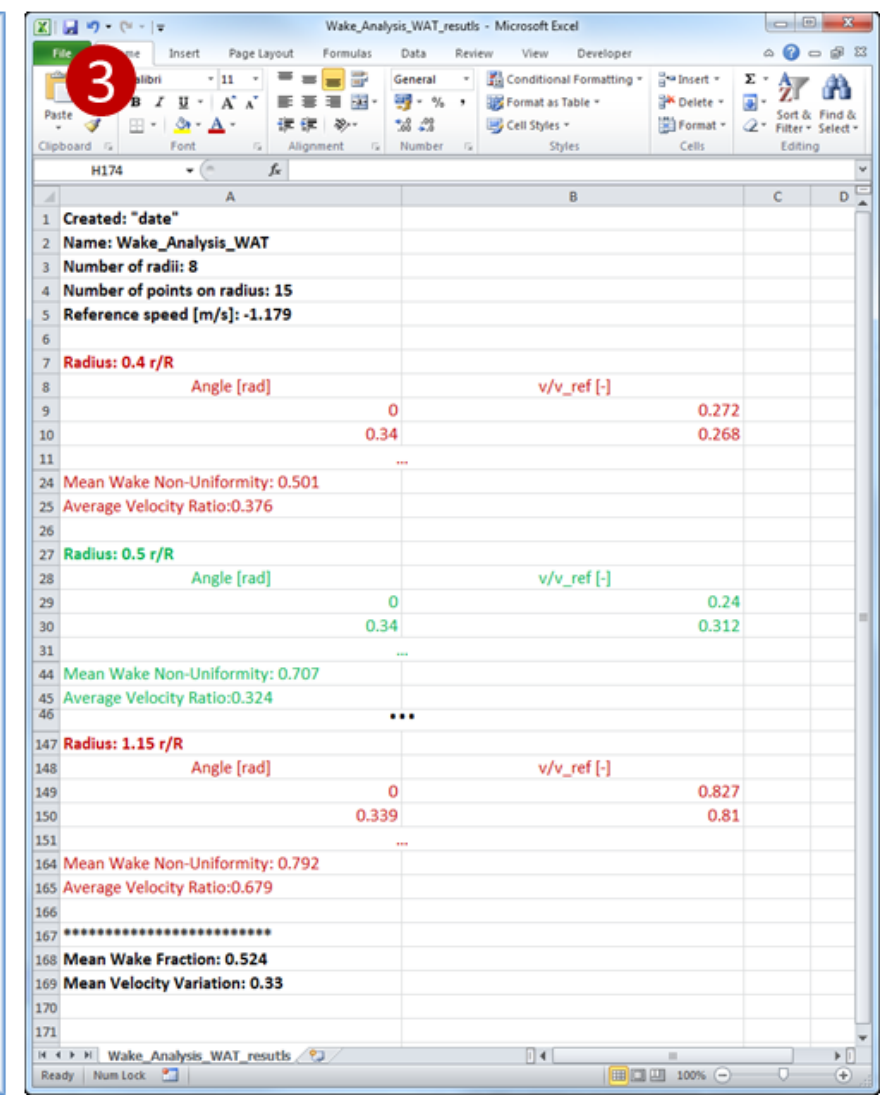

Figure 4 - Wake Analysis Tool (WAT) output 


\section{WAKE QUALITY ASSESSMENT EXTENSION}

Other than the standard wake analyses, another important measure to evaluate the wake quality is to use established wake criteria that also focus on hull-propeller interaction. This gives a means to measure and compare different wakes, allowing them to be classified as acceptable or unsatisfactory, together with the possibility of identifying the best design candidates.

Although empirical/statistical wake analyses are simplified methods they are quite popular in earlystage ship design procedures which are considered as initial indicators for a good or bad design. Various empirical wake criteria have been developed over the years. A more sophisticated analysis, published as B.S.R.A. (The British Ship Research Association) criteria, were developed by Odabasi and Fitzsimmons [7] who extended Huse's [6] work to develop criteria to assess for any potential propulsion or vibration problems. Due respect to the importance of the tangential wake velocity component for some aft end cases, it is good to note that, similar to the above presented standard wake analysis, these criteria also consider the wake velocities in the axial direction and exclude the components in the other directions. This was deemed appropriate on the basis that the axial component is considered the most dominant component. Odabasi and Fitzsimmons propose five wake quality criteria which mostly focus on the analysis of the top dead centre region (see Figure 2), defined by the angular interval $\varphi_{B}$ of the measured wake field. The WAT was extended to analyse the wake criteria by [7] which are defined as follows.

B.S.R.A. Criterion I - The first criterion states that within the angular interval $\varphi_{B}$ and in the range of fractional radius $\frac{r}{R}=0.4-1.15$, the maximum measured wake $w_{\max , \varphi_{B}}$ (with $w=1-\frac{v_{a}}{v}$ ) should be smaller than either 0.75 or the ship block coefficient $c_{B}$, whichever is smaller (5).

$$
w_{\max , \varphi_{B}}<\left(0.75, c_{B}\right)_{\min }
$$

B.S.R.A. Criterion II - The second criterion states, that the maximum acceptable wake peak on the measured wake disc $w_{\text {max,disc }}$ should not exceed $170 \%$ of the measured average wake at the effective non-dimensional propeller radius $\frac{r}{R}=0.7$ (6).

$$
w_{\text {max,disc }}<1.7 \bar{w}_{0.7}
$$

B.S.R.A. Criterion III - The third criterion analyses the width of the wake peak and relates it to the width of the wake shadow area. After analysing whether the measured wake distribution at the nondimensional propeller radius $\frac{r}{R}=1.0$ consists of a single wake peak with its maximum at $\varphi=0^{\circ}$ or of a double weak peak (see Figure 5) the width of the peak gets measured by finding the intersection of the wake minimum and the maximum absolute wake gradients before and after the peak. 


\section{Wake}

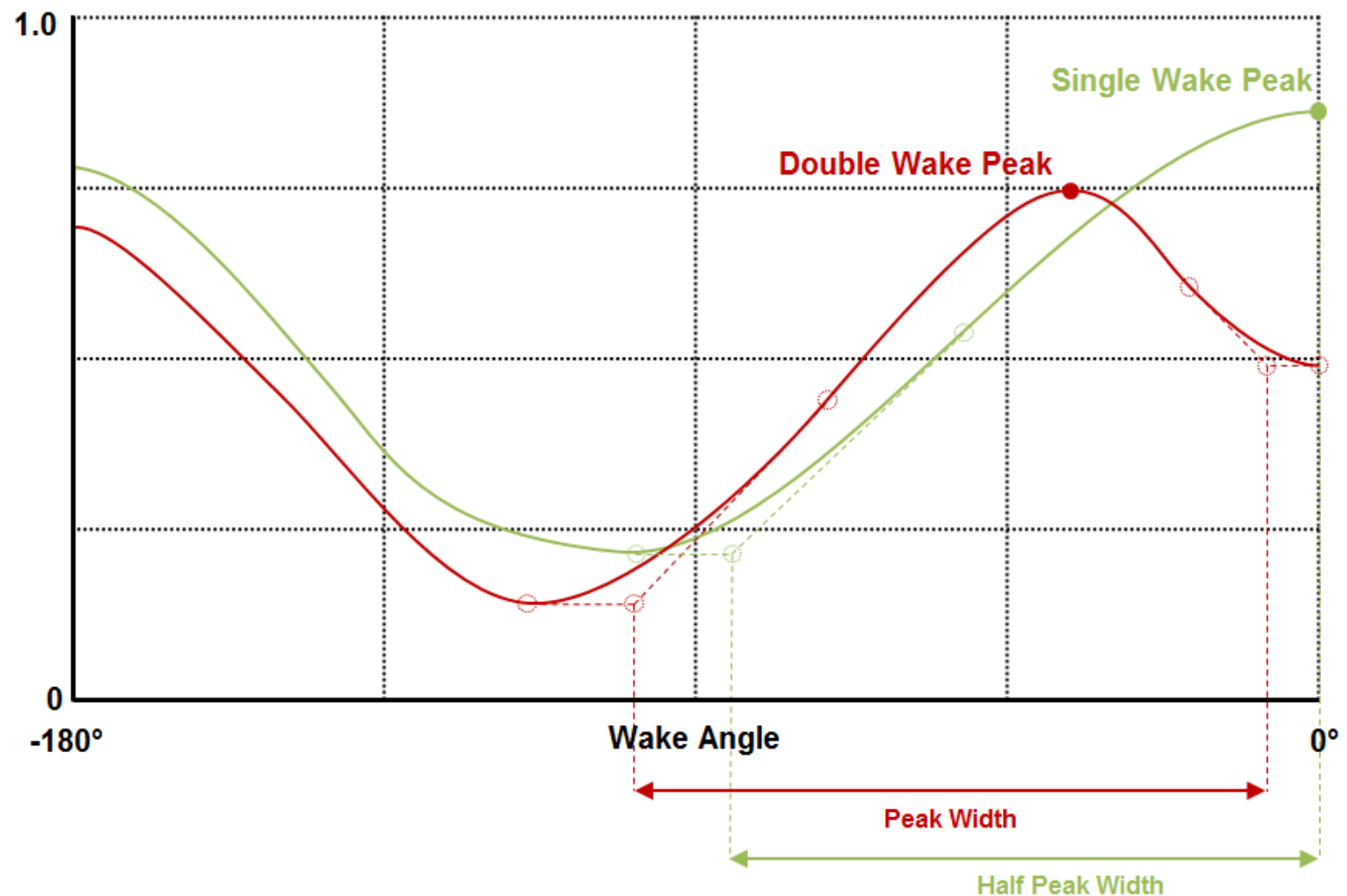

Figure 5 - Single and double wake peak width definition

Hereby the width of the measured peak should be larger than the wake shadow area to avoid high gradients within the wake shadow area.

$$
\varphi_{B}>\left(\varphi_{\max }\right)_{1.0}
$$

B.S.R.A. Criterion IV - The fourth criterion estimates the excitation forces on the hull by relating the tipspeed based cavitation number (8) and the wake non-uniformity (11) at the non-dimensional propeller radius $\frac{r}{R}=1.0$. The tip speed based cavitation number is defined as follows:

$$
\left(\sigma_{n I}\right)_{1.0}=\frac{P_{a}-P_{v}+P_{I}}{0.5 \rho(\pi N D)^{2}}
$$

$$
\begin{array}{lll}
\text { with } P_{I}=\rho g H_{i} & \text { (9) } \quad \text { and } \quad H_{i}=T_{A}-\left(\frac{D}{2}+Z_{p}\right)
\end{array}
$$

The corresponding intersection point of the wake non-uniformity $\left(w_{\Delta}\right)_{1.0}$ and the cavitation number $\left(\sigma_{n I}\right)_{1.0}$ should lie above the dividing band in Figure 6, originally provided by Odabasi and Fitzsimmons. The non-dimensional averaged wake non-uniformity $\left(w_{\Delta}\right)_{r}(11)$ gives a measure for the extent of the velocity variation on each radius.

$$
\left(w_{\Delta}\right)_{r}=\frac{\Delta(w)_{r}}{1-(\bar{w})_{r}}
$$


The maximum wake variation $\Delta w(12)$ and the average wake $\bar{w}$ on each radius (13) are defined as follows.

$$
\begin{gathered}
\Delta(w)_{r}=\left(1-\frac{v_{a}}{v}\right)_{r, \max }-\left(1-\frac{v_{a}}{v}\right)_{r, \min } \\
(\bar{w})_{r}=\left(1-\frac{\overline{v_{a}}}{v}\right)_{r}
\end{gathered}
$$

Tip Cavitation Number

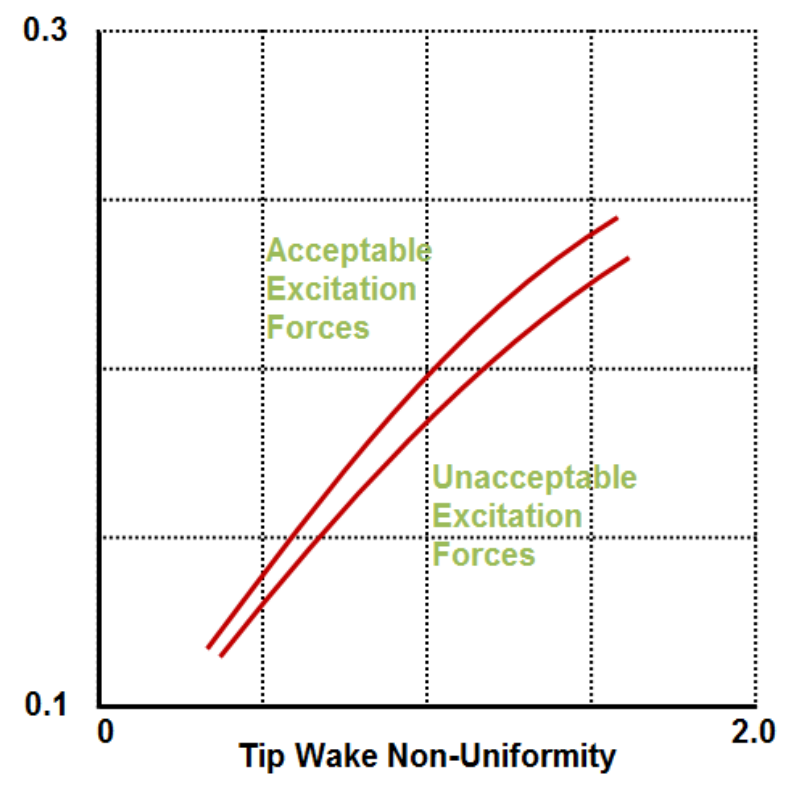

Figure 6 - Wake non-uniformity criterion

B.S.R.A. Criterion $V$ - The fifth criterion only needs to be checked if the fourth criterion returns an intersection point that falls within the dividing band of Figure 6 . This would indicate that the propeller is susceptible to cavitation. In this case the local wake gradient $\frac{d w}{\mathrm{~d} \varphi}$ per unit axial velocity for all measured radii $\frac{r}{R}$ inside the angular interval $\varphi_{B}$ in the range of $\frac{r}{R}=0.7-1.15(14)$ should be less than one.

$$
\frac{1}{r / R}\left|\frac{d w / \mathrm{d} \varphi}{(1-w)}\right|<1.0
$$

After having integrated the above outlined B.S.R.A. criteria into the WAT code, the graphical output (see Figure 7) reflects the analysis outcome by stating whether or not the criteria were fulfilled (\#5). In addition the output shows the extend of the BRSA wake shadow angle added to the top plot (\#1), a detailed plot for the third criterion (\#2) and the excitation force estimation plot for the fourth criterion (\#4). The bottom-left plot (\#3) remained unchanged (compared to Figure 4 (\#1)). 

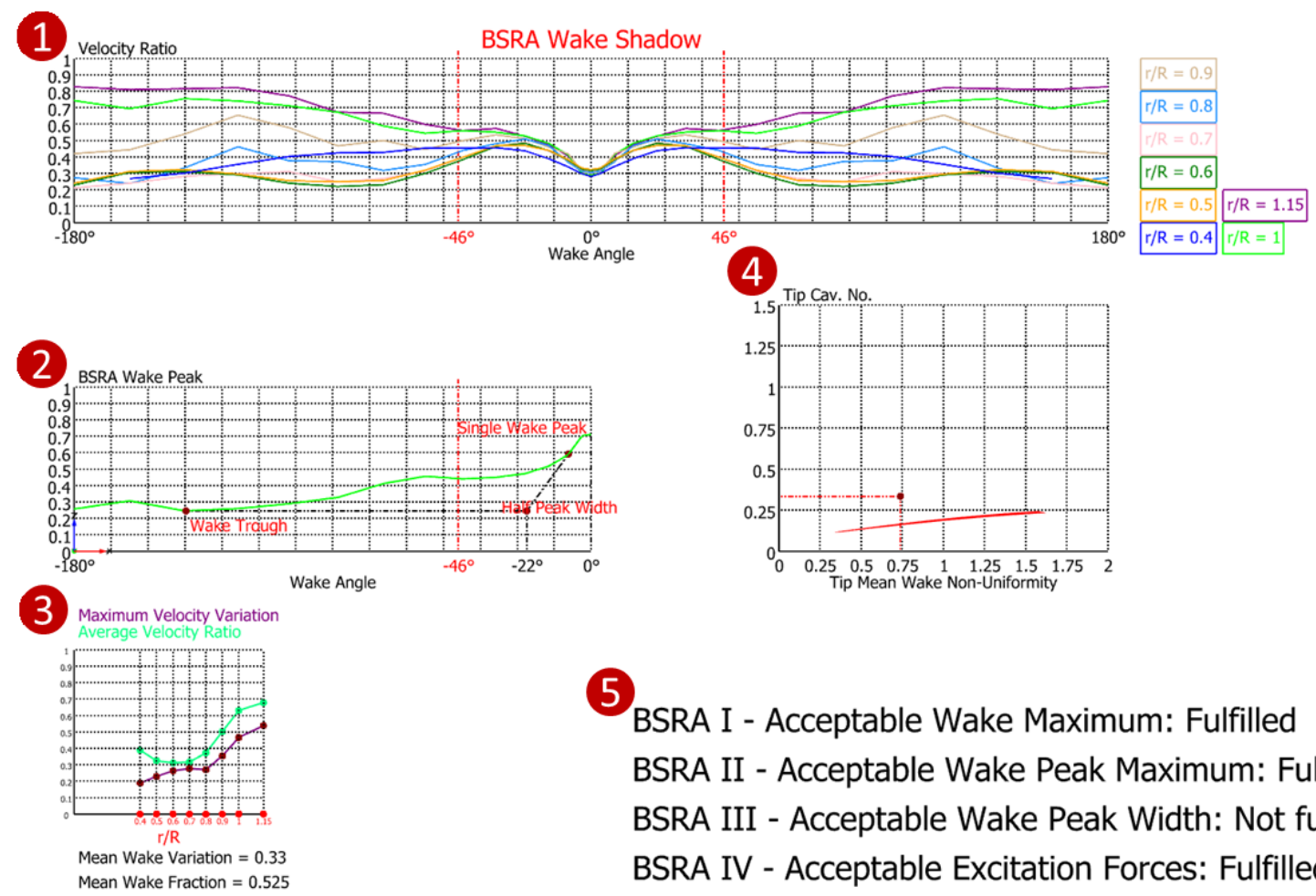

5 BSRA I - Acceptable Wake Maximum: Fulfilled BSRA II - Acceptable Wake Peak Maximum: Fulfilled BSRA III - Acceptable Wake Peak Width: Not fulfilled BSRA IV - Acceptable Excitation Forces: Fulfilled

Figure 7 - Visual output

\section{PRE-PROCESSING AUTOMATION}

As already mentioned, the developed WAT can be used as a numerical post-processing tool to evaluate the quality of a propeller wake field. In order to further automate the CFD pre-processing as well, the presented case study also includes the custom generation of the required CSV input file.

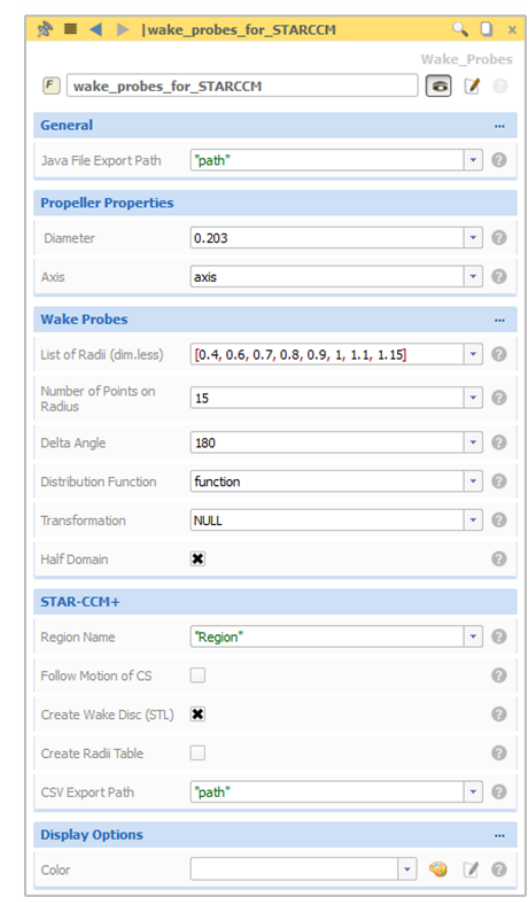

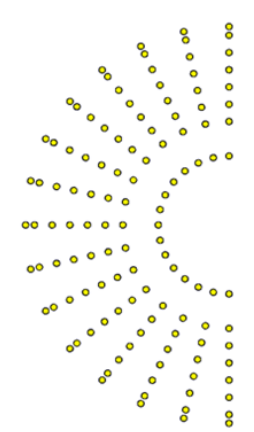

Linear Distribution

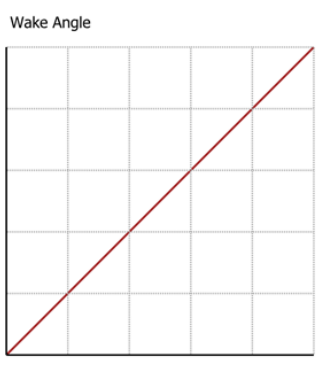

Points Distribution along Radius

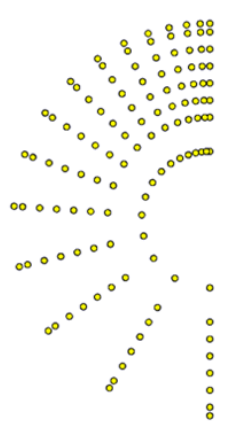

Distribution concentratedin Wake Shadow

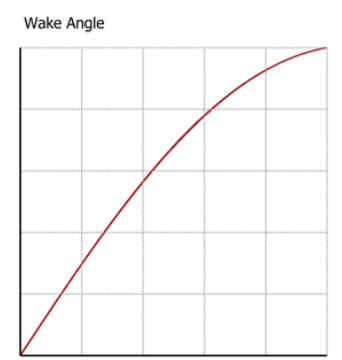

Points Distribution along Radius

Figure 8 - Wake Probe Code Interface (LHS) and wake probe samples using different distribution functions (RHS) 
The first step of the process makes use of an additional feature code that creates a user-defined JAVA Script. This triggers the generation of the wake CSV file by generating and evaluating the necessary objects of the CFD simulation setup. Figure 8 shows the code interface along with a standard linear points-distribution and a custom distribution that concentrates the probe points in the top dead centre of the propeller plane (wake shadow area).

It has to be noted that the pre-processing tool is tailored to suite the requirements of the specific numerical solver and is therefore not of universal use. However, the integration shows that it is possible to automate the wake analysis pre-processing for a commercial tool and it is assumed that a similar approach can be used for other flow solvers. With the above introduced pre-processing tool the software chain was fully established (see again Figure 3 ) and ready for automated design variation and optimisation studies. 


\section{CFD Case Study}

The aim of this study was to demonstrate the usefulness of the developed Wake Analyses Tool (WAT) by applying it to a set of nominal ship wake fields that were generated by performing CFD simulations. It was therefore deemed appropriate to use the open source cape-size bulk carrier called Japan Bulk Carrier (JBC) that is alternatively provided with an installed Energy Saving Device. The ship geometry was used for the workshop on CFD in ship hydrodynamics in Tokyo [20]. The JBC hull and its appended circular duct have been recently designed by National Maritime Research Institute (NMRI), Yokohama National University and Ship Building Research Centre of Japan (SRC).

A set of experimental data is available. This includes the measurement of nominal parameters such as the hull resistance and the contour of the nominal wake field at different transversal planes. Due to the high block coefficient $\left(c_{B}\right)$, towing tank experiments including wake field measurements have shown that the JBC produces rather challenging operating conditions for a propeller. The nominal propeller wake field consists of areas of stagnated flow and a pronounced wake shadow characteristic. Experimental results have also shown that the JBC design with duct produces more favourable selfpropulsion conditions. The experiments, however, do not provide a prediction of levels of vibration induced by the rotating propeller. In order to estimate such effects, the implemented B.S.R.A. criteria were applied to the calculated wake fields.

For this study, in order to create a set of wake fields, 4 different operating conditions were simulated for both the hull without duct and with duct. The presented variation study was carried out in model scale and compared to the experimental results for the design conditions. Table 1 provides an overview of all simulated conditions defined by a lower draft and a lower speed compared to design conditions.

Table 1 - Draft and speed conditions for variation study

\begin{tabular}{|c|c|c|c|c|}
\hline Design & \multicolumn{2}{|c|}{ Baseline } & \multicolumn{2}{c|}{ Duct } \\
\hline Speed $\left(\frac{m}{s}\right)$ & $\mathbf{1 . 1 7 9}(\mathbf{1 4 . 5} \mathbf{~} n)$ & $\mathbf{0 . 8 1 3 1}(\mathbf{1 0} \mathrm{kn})$ & $\mathbf{1 . 1 7 9}(\mathbf{1 4 . 5} \mathrm{kn})$ & $\mathbf{0 . 8 1 3 1}(\mathbf{1 0} \mathrm{kn})$ \\
\hline Draft $(\boldsymbol{m})$ & $\begin{array}{c}B L_{I}(\text { Design } \\
\text { Condition })\end{array}$ & $B L_{I I}$ & $\begin{array}{c}D_{I} \text { (Design } \\
\text { Conditions) }\end{array}$ & $D_{I I}$ \\
\hline $\mathbf{0 . 4 1 2 5}(16.5 \mathrm{~m})$ & $B L_{I I I}$ & $B L_{I V}$ & $D_{I I I}$ & $D_{I V}$ \\
\hline $\mathbf{0 . 3 6 2 5}(14.5 \mathrm{~m})$ & & \\
\hline
\end{tabular}

${ }^{*}$ Corresponding full-scale conditions in red font

\section{GEOMETRY}

This high block coefficient vessel was intentionally chosen due to its complex stern flow characteristics making it a suitable candidate for research. Experimental tests of the JBC were purposely built to serve as a benchmark database for detailed flows around a ship with an energy saving device (see Figure 9).

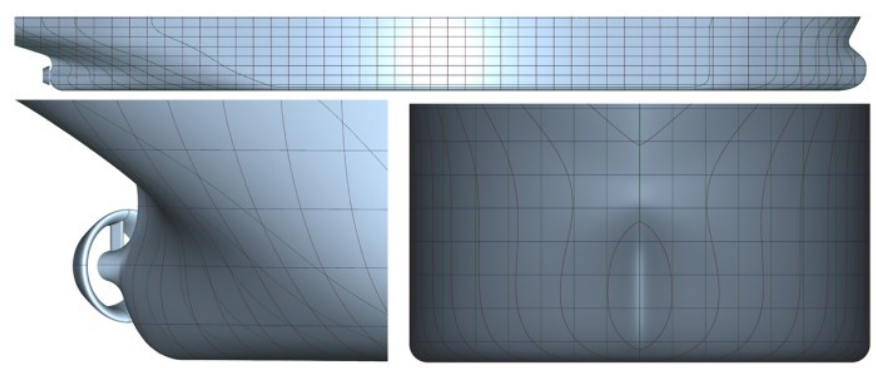

Figure $9-\mathrm{JBC}$ hull geometry 
NMRI carried out experimental tests in their towing tank measuring the resistance, self-propulsion parameters as well as detailed velocity distribution around the stern for the JBC with and without the circular duct. Table 2 shows the main particulars of both ship designs.

Table 2 - Japan Bulk Carrier (JBC) Particulars

\begin{tabular}{|l|c|c|c|}
\hline \multicolumn{2}{|c|}{ Particulars } & Full Scale & Model Scale \\
\hline Model scale factor & $\lambda$ & 1 & 40 \\
\hline $\begin{array}{l}\text { Length between } \\
\text { perpendiculars }\end{array}$ & $\mathrm{LPP}(\mathrm{m})$ & 280 & 7.0 \\
\hline Length of waterline & $\mathrm{LWL}(\mathrm{m})$ & 285 & 7.1250 \\
\hline $\begin{array}{l}\text { Maximum beam of } \\
\text { waterline }\end{array}$ & $\mathrm{BWL}(\mathrm{m})$ & 25 & 1.1250 \\
\hline Depth & $\mathrm{D}(\mathrm{m})$ & 16.5 & 0.6250 \\
\hline Draft & $\mathrm{T}_{\mathrm{A}}(\mathrm{m})$ & 178369.9 & 0.4125 \\
\hline Displacement volume & $\nabla\left(\mathrm{m}^{3}\right)$ & 19556.1 & 2.7870 \\
\hline $\begin{array}{l}\text { Wetted surface area w/o } \\
\text { ESD }\end{array}$ & $\mathrm{S}_{\mathrm{Baseline}}\left(\mathrm{m}^{2}\right)$ & 19633.9 & 12.2206 \\
\hline $\begin{array}{l}\text { Wetted surface area with } \\
\text { ESD }\end{array}$ & $\mathrm{S}_{\mathrm{ESD}}\left(\mathrm{m}^{2}\right)$ & 0.858 & 12.2696 \\
\hline Block coefficient & $\mathrm{c}_{\mathrm{B}}$ & 0.8580 \\
\hline
\end{tabular}

NUMERICAL SOLVER

A commercial flow solver was used to model the multiphase flow using Unsteady Reynold Averaged Navier-Stokes (URANS) equations to simulate a three-dimensional environment using a two-equation eddy viscosity model. In particular, the Shear Stress Transport (SST) k- $\omega$ model with Curvature Correction was used. The continuity and momentum governing equations [21] for incompressible flows can be represented in tensor notation and Cartesian coordinates as follows:

$$
\begin{gathered}
\frac{\partial\left(\rho \overline{\mathrm{u}}_{\mathrm{i}}\right)}{\partial \mathrm{x}_{\mathrm{i}}}=0 \\
\frac{\partial\left(\rho \overline{\mathrm{u}}_{\mathrm{i}}\right)}{\partial \mathrm{t}}+\frac{\partial}{\partial \mathrm{x}_{\mathrm{j}}}\left(\rho \overline{\mathrm{u}}_{\mathrm{i}} \overline{\mathrm{u}}_{\mathrm{j}}+\rho \overline{\mathrm{u}_{\mathrm{i}} \dot{\mathrm{u}}_{\mathrm{j}}}\right)=-\frac{\partial \overline{\mathrm{p}}}{\partial \mathrm{x}_{\mathrm{i}}}+\frac{\partial \overline{\mathrm{\tau}}_{\mathrm{ij}}}{\partial \mathrm{x}_{\mathrm{j}}} \\
\overline{\mathrm{\tau}}_{\mathrm{ij}}=\mu\left(\frac{\partial \overline{\mathrm{u}}_{\mathrm{i}}}{\partial \mathrm{x}_{\mathrm{j}}}+\frac{\partial \overline{\mathrm{u}}_{\mathrm{j}}}{\partial \mathrm{x}_{\mathrm{i}}}\right)
\end{gathered}
$$

With regards to the air-water interface capturing scheme, a Volume of Fluid (VOF) method was used to model the free surface effects using a second order convection scheme. The VOF method computed the volume fraction of the immiscible fluids (air and water) to predict the motion of the free surface. The flow solver employed the SIMPLE velocity-pressure coupling algorithm in a segregated manner using a pressure correction equation to fulfil mass conservation. All the case studies were carried out in calm water conditions. The vessel was allowed to pitch and heave using the Dynamic Fluid Body Interaction (DFBI) feature that computed the forces and moments on the vessel solving the governing equations of rigid body motion. A hybrid wall treatment approach was used with an appropriate blending of the prism layer cells to the near domain cells.

For the VOF free surface prediction and the DFBI motions computation to work, an implicit unsteady time marching scheme along with a Finite Volume Method (FVM) approach was carried out to treat temporal and spatial discretization. The time step $\Delta t=0.025 \mathrm{~s}$ was selected as to give Courants numbers (CFL) $C F L \approx 1$ for numerical stability. Although the simulations were solved in time, global 
phenomena, such as the free surface wave pattern and ship motions were expected to reach a quasisteady state.

The volume mesh was generated using an automatic mesh grid generation tool that makes use of a Cartesian cut-cell method also known as the Trimmer. An unstructured grid of around 6.8 million hexahedral cells was produced. Mesh refinements were specified in designated areas of interest, particularly the stern region for accurate prediction of stern flow behaviour (see LHS Figure 10).
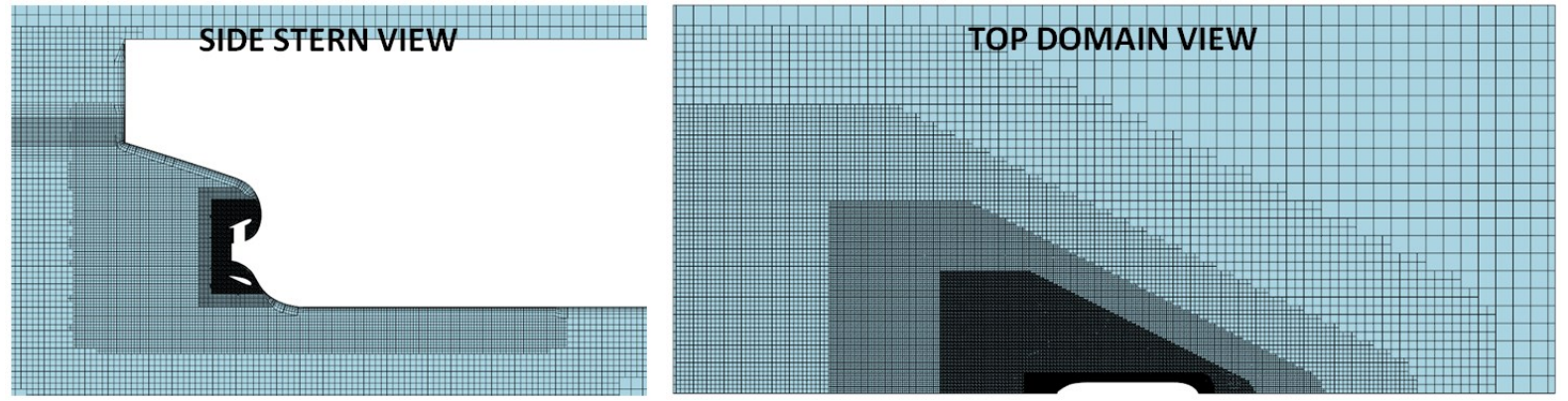

Figure 10 - Mesh refinements

Despite the variations in the volume mesh due to the mesh refinements, it was made sure that there was reasonable uniform growth between cells avoiding high skew developments. To keep the number of cells as low as possible free surface refinements were mostly restricted to the ship wake field. Figure 10 gives an impression of the mesh in the stern region (LHS) and the free surface region (LHS) showing the typical refinement shape, following the Kelvin angle, creating a dense mesh near the hull and getting coarser far away from the hull. In the field of Computational Fluid Dynamics, the cells nearer the surface/wall boundaries are also of prime concern. Fluid flow passing along a surface generates a boundary layer that develops due inertia forces and viscous forces producing different turbulent. In order to accurately predict near wall flow details, a prism layer mesh was employed, generating 5 orthogonal prismatic cells adjacent to the surface along the ship hull. It is good to note that the prism layer thickness was varied along the hull constructing a nice blend to the nearest core cells that resulted due to different mesh refinements. The "All $y+$ Wall Treatment" model was selected for the simulation with the prism layer mesh designed to give an average $y+\approx 35$. This ensured appropriate treatment of the near wall flow using the wall-function approach. Figure 11 shows both the distribution of $y+$ on the underwater hull cells and a contour plot. For a smaller number of cells $y+$ is smaller than 30 which indicates flow regions of stagnation or separation (example shown in Figure 15).

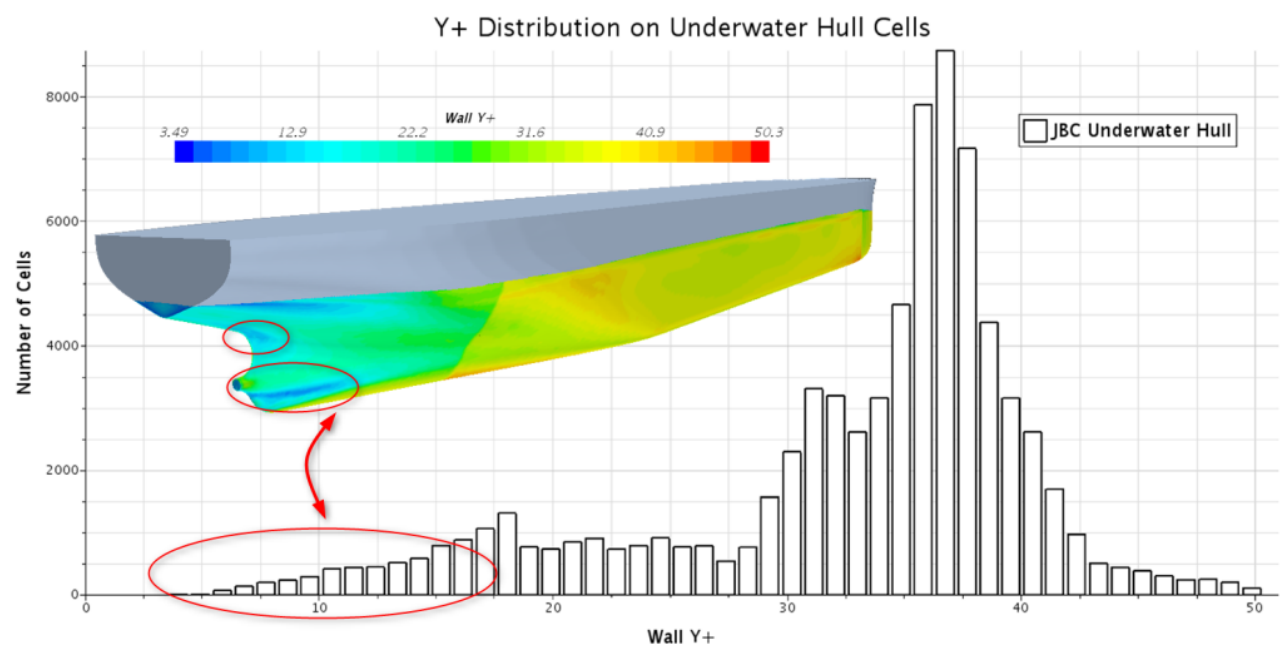

Figure 11 - Exemplary Wall $\mathrm{Y}+$ values of underwater hull 
The appropriate choice of boundary conditions is of critical importance ensuring the right methodology and approach of a numerical simulation. Suitable initial and boundary conditions have an influence on faster time convergence that can save on computational cost [22]. The same authors continue to add that the distance and positioning of the boundary conditions are equally important. Both should be defined in such a way that the boundaries have no influence on the flow characteristics/ behaviour under investigation resulting in boundary independent solutions. A velocity flow field condition was specified at the inlet boundary located 2.5 ship lengths upstream of the vessel and a pressure field for the outlet boundary placed $2.8 \mathrm{Lpp}$ downstream. Since the conditions of a towing test simulation are symmetrical about the centre plane (along the keel), it was deemed feasible to model half the domain. A symmetry plane was thus set along the x-axis. The hull geometry was specified with a non-slip wall boundary allowing boundary layer developments. On the other hand, the remaining boundaries were set to velocity inlet conditions featuring slip wall characteristics thus preventing the development of velocity gradients between the wall and fluid. The bottom boundary was placed far enough below the water level to avoid any shallow water effects. VOF wave damping was also applied to the inlet, outlet and side boundaries preventing wave reflections that might interfere with the results.

\section{VALIDATION AND VERIFICATION}

Validation and verification $(\mathrm{V} \& \mathrm{~V})$ procedures for the model scale virtual towing tests were carried out using the open source JBC hull geometry. For this particular study, the V\&V procedure followed the methodology presented by Xing and Stern [19]. The simulation error $\delta_{S}$ is a combination of modelling and numerical errors. In a similar manner, the simulation uncertainty is also a combination of modelling and numerical uncertainties. The validation process provided the modelling uncertainty $U_{S M}$ and error $\delta_{S M}$ whereas the verification procedure identified the numerical uncertainty $U_{S N}$ and numerical errors $\delta_{S N}$ demonstrating the capability of the solver.

A grid convergence study was carried out to investigate the influence of an increasing mesh resolution on the solver solution. Such a study requires at least three solutions by varying the numerical mesh size. For that purpose, three grids were systematically generated by varying the cell size in the domain by a factor of $\sqrt{2}$ in each spatial direction. That being said, the first prism layer height on the wall was retained constant across all meshes preventing any changes of the wall treatment of the turbulence model. All three grids (Fine, Medium and Coarse) were computed producing three solutions $S_{1}, S_{2}, S_{3}$ respectively, allowing the derivation of the convergence ratio.

In marine CFD simulations it is common practice to validate and verify the total ship drag coefficient $c_{T}$ along with the ship motions, here dynamic sinkage and dynamic trim. The convergence ratio for the grid dependency study exhibited a monotonic convergence according to the criteria specified by [19]. The numerical errors and uncertainties were then computed using the Generalised Richards Extrapolation $(R E)$ originally developed by [23] and further adapted by [19] to account for higher-order terms. Table 3 presents the different solutions achieved for the grid densities indicating minor and comparable error percentages. In all computed cases, the free surface, the ship motions and hence the ship total resistance converged to quasi-steady values.

Table 3: Grid Dependency Study for $c_{T}$, sinkage and trim

\begin{tabular}{lccc}
\hline & Coarse & Medium & Fine \\
\hline Base Size $(m)$ & 0.22 & 0.156 & 0.11 \\
Mesh Size $(M)$ & 1.36 & 2.7 & 6.87 \\
$c_{T}$ Error \% & 2.85 & 2.29 & 2.28 \\
Sink/Lpp error \% & -3.51 & -2.02 & -1.62 \\
Trim/Lpp error\% & -4.20 & -3.96 & -3.86 \\
\hline
\end{tabular}


The verification of the drag coefficient for the grid and time step convergence study yielded insignificant uncertainties as presented in Table 4 for the different methods indicating that the results are not sensitive or dependant on the grid size/time step when using the fine mesh. The order of accuracy for the grid study and the time step study resulted in $p_{G}=8.4606$ and $p_{T}=4.5912$. The estimate for the limiting order of accuracy was set to $p_{e s t}=2$.

The numerical uncertainties were estimated using Roaches' [24] Grid Convergence Index GCI with a factor of safety $(F S=1.25)$ as recommended by Celik [25]. Alternatively, $R E$ with the concept of correction factors was also used to estimate the errors and uncertainties [19]. The extended correction factor to the Richards extrapolation is a means to determine the proximity of solution to the asymptotic range.

Table 4 : Grid and Time Step Verification Study for $C_{\mathrm{T}}\left({ }^{*} 10^{-3}\right)$

\begin{tabular}{|c|c|c|c|c|c|c|c|c|c|c|c|c|}
\hline \multirow{2}{*}{ Parameter } & \multirow{2}{*}{$E F D$} & \multirow{2}{*}{$S_{C}$} & \multirow{2}{*}{$r_{G / T}$} & \multicolumn{3}{|c|}{ Solutions } & \multirow{2}{*}{$\boldsymbol{R}_{G}$} & \multirow{2}{*}{$\boldsymbol{\delta}_{G}\left(\% \boldsymbol{S}_{1}\right)$} & \multicolumn{2}{|c|}{$U_{G / T}\left(\% S_{1}\right)$} & \multicolumn{2}{|c|}{$U_{G C / T C}\left(\% S_{1}\right)$} \\
\hline & & & & $S_{1}$ & $S_{2}$ & $S_{3}$ & & & $C F$ & $G C I$ & $C F$ & $G C I$ \\
\hline$C_{T}($ Grid $)$ & 4.289 & 4.1972 & $\sqrt{2}$ & 4.1914 & 4.1901 & 4.1657 & 0.0532 & -0.0310 & 0.0603 & 0.0021 & 0.0293 & 0.0004 \\
\hline $\begin{array}{c}C_{T} \text { (Time } \\
\text { Step) }\end{array}$ & 4.289 & 4.369 & $\sqrt{2}$ & 4.1914 & 4.2091 & 4.2960 & 0.2037 & 0.4223 & 0.7366 & 0.1350 & 0.3143 & 0.0270 \\
\hline
\end{tabular}

In the respective validation approach the comparison error $E$ is considered to be the difference between the solver output $S$ and the true value $T$. Deducting the experimental errorl estimate $\delta_{D}$ from the data $D$ gives $T$. $E$ is generally compared to the validation uncertainty $U_{v}$ in order to determine whether the numerical model complies. For this case study, the solver output is $C_{T}$ (drag coefficient) with $D$ being the experimental value and the experimental uncertainty given to be $1 \%$.

Table 5 : Grid and Time Step Validation for $C_{T}$

\begin{tabular}{|c|c|c|c|c|c|c|}
\hline \multirow{2}{*}{ Parameter } & \multicolumn{2}{|c|}{ Uncorrected } & \multirow{2}{*}{$U_{D}$} & \multicolumn{2}{|c|}{ Corrected } & \multirow{2}{*}{$\boldsymbol{E}(\%)$} \\
\hline & $U_{S N}$ & $\boldsymbol{U}_{v}$ & & $U_{S N}$ & $\boldsymbol{U}_{v}$ & \\
\hline$C_{T}($ Grid $)$ & 0.0589 & 1.0017 & 1.0 & 0.0286 & 1.0 & 2.2453 \\
\hline $\begin{array}{c}C_{T} \text { (Time } \\
\text { Step) }\end{array}$ & 0.7198 & 1.2321 & 1.0 & 0.3071 & 1.0461 & 2.6883 \\
\hline
\end{tabular}

The grid comparison error $|E|$ shows a simulation accuracy of $2.25 \%$ for the total drag coefficient $C_{T}$ being higher than the experimental uncertainty (see Table 5). The iterative uncertainty was assessed by calculating half the range of the maximum and minimum values from the $c_{T}$ mean value over the last three periods of oscillation (corresponds to $10 \mathrm{~s}$ of solution time) which yielded an iterative uncertainty of $U_{I}=0.6$. Commonly, the accuracy of experimental versus numerical wake fields is assessed by a visual comparison of contour plots such as shown in Figure 12. Although both wake fields look similar to some extend the above approach does not allow a detailed comparison. To perform a V\&V study for the wake field, the experimental wake field (EFD) was analysed on a point-by-point basis using the WAT and compared to the numerical predictions on a coarse, medium and fine mesh. Figure 13 shows the velocity ratios $v_{r i}$ for the dimensionless radii $\frac{\mathrm{r}}{\mathrm{R}}=0.7-1.0$ in steps of $\frac{\mathrm{r}}{\mathrm{R}}=0.1$ within the wake field shadow (top dead centre) on the LHS and the corresponding contour plot of the experimental wake field on the RHS. 


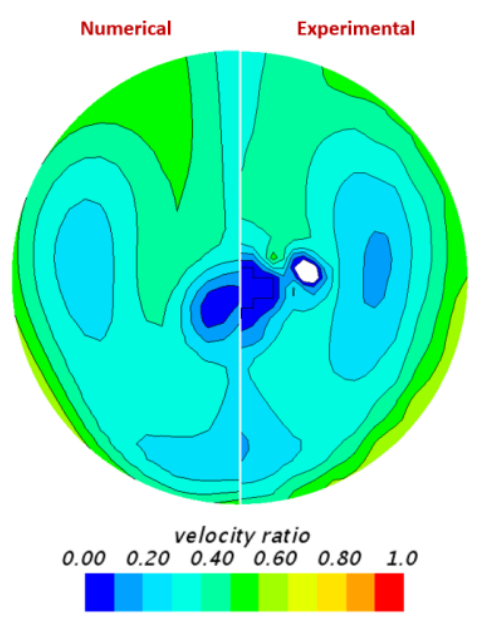

Figure 12 - Experimental and numerical wake field contour plot comparison

In the below figure, red points represent the extraction points, starting from a wake angel of $46^{\circ}$ going up in a non-constant manner to the top position of the wake disc.
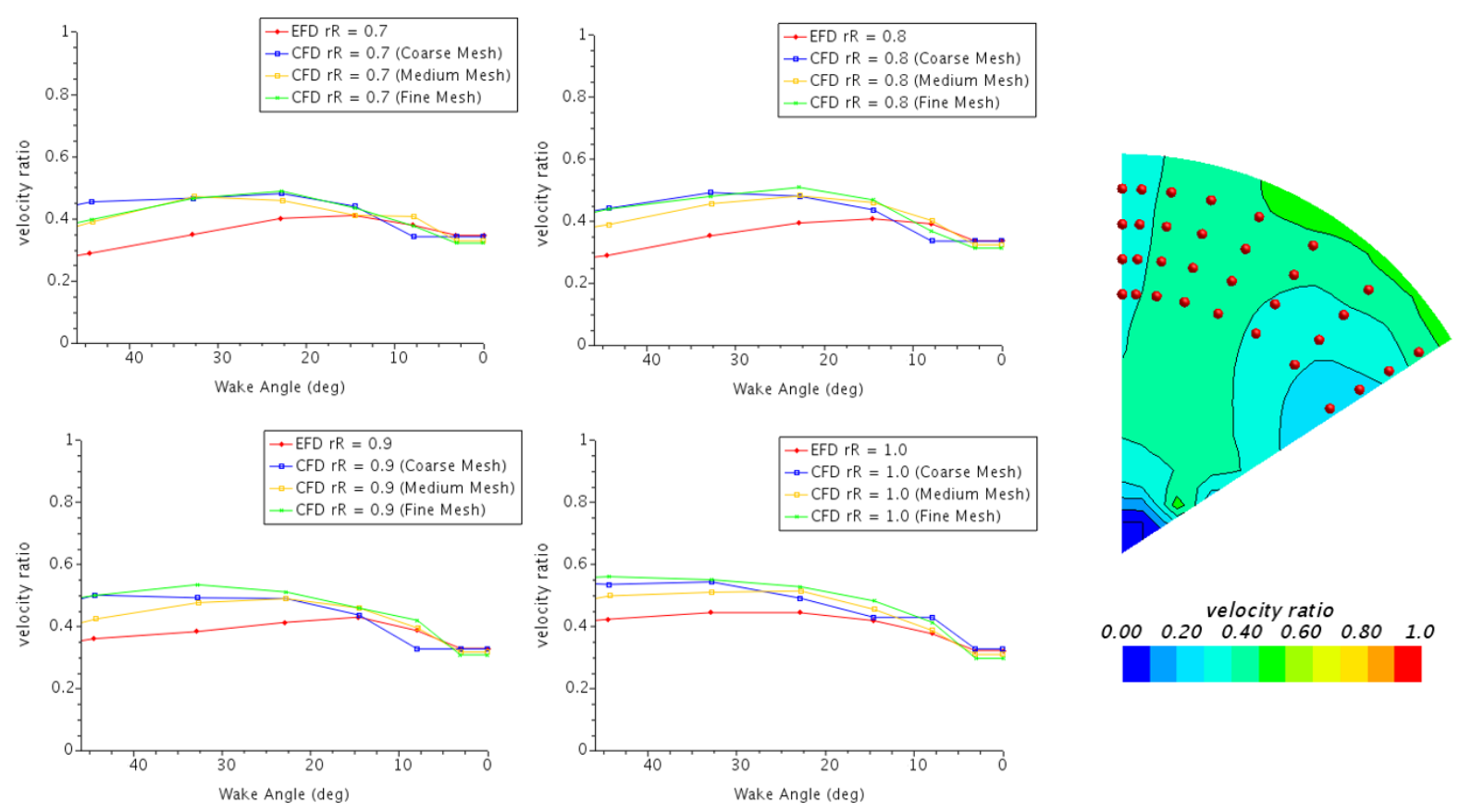

velocity ratio

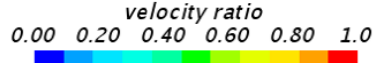

Figure 13 - Experimental and numerical wake field comparison

For the radius $\frac{r}{R}=0.7$ an attempt was made to use the point data to calculate the V\&V errors and uncertainties. However, as shown in Table 6 , most data points showed oscillatory convergence $\left(R_{G}<\right.$ 0 ). In this study, the wake field was not recorded in a transient manner but extracted at the last time step assuming a quasi-steady state solution. Hence, upper and lower bounds of the solution oscillation could not be extracted and the iterative uncertainty could not be calculated. 
Table 6 - Grid Convergence Study for wake field radius $\frac{r}{R}=0.7$

\begin{tabular}{|c|c|c|c|c|c|c|c|c|c|c|c|c|}
\hline \multirow{2}{*}{ Parameter } & \multirow{2}{*}{$E F D$} & \multirow{2}{*}{$S_{C}$} & \multirow{2}{*}{$r_{G}$} & \multicolumn{3}{|c|}{ Solutions } & \multirow{2}{*}{$\boldsymbol{R}_{G}$} & \multirow{2}{*}{$\delta_{G}\left(\% S_{1}\right)$} & \multicolumn{2}{|c|}{$U_{G}\left(\% S_{1}\right)$} & \multicolumn{2}{|c|}{$U_{G C}\left(\% S_{1}\right)$} \\
\hline & & & & $S_{1}$ & $S_{2}$ & $S_{3}$ & & & $C F$ & GCI & $C F$ & $G C I$ \\
\hline$v_{r 1}$ & 0.288 & N/A & $\sqrt{2}$ & 0.397 & 0.390 & 0.454 & -0.11 & --- & - & --N/A-- & - & ------ \\
\hline$v_{r 2}$ & 0.349 & $\mathrm{~N} / \mathrm{A}$ & $\sqrt{2}$ & 0.465 & 0.471 & 0.466 & -1.2 & $\ldots$ & 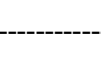 & --N/A--- & 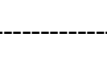 & ---- \\
\hline$v_{r 3}$ & 0.401 & $\mathrm{~N} / \mathrm{A}$ & $\sqrt{2}$ & 0.488 & 0.458 & 0.480 & -1.36 & --- & 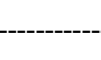 & --N/A--- & - & ------- \\
\hline$v_{r 4}$ & 0.411 & N/A & $\sqrt{2}$ & 0.434 & 0.411 & 0.440 & -0.79 & ----- & 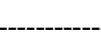 & --N/A--. & 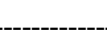 & ---- \\
\hline$v_{r 5}$ & 0.379 & $\mathrm{~N} / \mathrm{A}$ & $\sqrt{2}$ & 0.377 & 0.407 & 0.342 & -0.46 & --- & (2) & $--\mathrm{N} / \mathrm{A}--$ & & ----- \\
\hline$v_{r 6}$ & 0.347 & 0.315 & $\sqrt{2}$ & 0.322 & 0.329 & 0.342 & 0.54 & 2.1739 & 3.2609 & 3.1703 & 0.3778 & 0.6341 \\
\hline$v_{r 7}$ & 0.347 & 0.315 & $\sqrt{2}$ & 0.322 & 0.329 & 0.342 & 0.54 & 2.1739 & 3.2609 & 3.1703 & 0.3778 & 0.6341 \\
\hline
\end{tabular}

Only the last two data points showed monotonic convergence which allowed to derive uncertainties and errors. Table 7 shows the grid validation results for those data points yielding a high grid comparison error $|E|$ of around $9 \%$ with an order of accuracy of $p_{G}=1.7862$. Again, the estimate for the limiting order of accuracy was set to $p_{e s t}=2$. Although the experimental uncertainty for the wake measurements was not given, again $1 \%$ was assumed to be realistic given the fact that an accurate PIV measurement method was used. A time step study was only performed to evaluate the dependency of the total resistance coefficient, hence the time step uncertainty with regard to the wake field solution was not calculated.

Table 7 - Grid Validation for wake field radius $\frac{r}{R}=0.7$

\begin{tabular}{cccccccc}
\hline \multirow{2}{*}{ Parameter } & \multicolumn{2}{c}{ Uncorrected } & \multirow{2}{*}{$\boldsymbol{U}_{\boldsymbol{D}}$} & \multicolumn{2}{c}{ Corrected } & \multirow{2}{*}{$\boldsymbol{E}(\%)$} \\
\cline { 2 - 3 } & $\boldsymbol{U}_{\boldsymbol{S N}}$ & $\boldsymbol{U}_{\boldsymbol{v}}$ & & $\boldsymbol{U}_{\boldsymbol{S N}}$ & $\boldsymbol{U}_{\boldsymbol{v}}$ & \\
\hline$v_{r 6}(\mathrm{Grid})$ & 3.0259 & 3.1869 & 1.0 & 0.3506 & 1.0597 & 9.2219 \\
\hline$v_{r 7}($ Grid) & 3.0259 & 3.1869 & 1.0 & 0.3506 & 1.0597 & 9.2219 \\
\hline
\end{tabular}

For further understanding and detailed information on the above analyses, reference can be made to [19]. 


\section{Results \& Discussion}

Before running the different operating conditions using both the Japan Bulk Carrier (JBC) baseline design and the JBC duct design, both design variants were firstly evaluated at their design conditions to evaluate the difference in resistance and stern flow characteristics. This was done in order to show that the calculated flow fields agree with the experimental data. The Wake Analysis Tool (WAT) was then applied to analyse each design variants wake field for its quality, i.e. mean wake variation and mean wake fraction, and whether or not the above outlined B.S.R.A. criteria were fulfilled.

\section{RESISTANCE \& STERN FLOW EVALUATION}

In order to analyse the differences that result when simulating both JBC designs at the same operational condition it is worthwhile to outline the theoretical and practical impact of a duct on the flow regime. The $\mathrm{JBC}$ pre-duct is a flow conditioning device that is mounted upstream of the propeller aiming to improve the propulsive efficiency of the vessel.Terwisga [26] explains that the working mechanism of the preduct in self-propulsion conditions is to increase the mass flow through the propeller, reducing the wake non-uniformity and the flow separation at the aft end of ship hulls that are subject to heavy separation of flow. Having not yet carried out self-propulsion simulations for this particular study, it is not possible to directly investigate the impact of the duct on the propulsion characteristics. That being said, the nominal towing test simulations are able to provide an indication of the reduction in resistance that would in turn affect the thrust deduction factor and the hull efficiency. Visonneau [9] outlines that this drag reduction is due to the suction effect of the duct that minimises the unsteadiness at the stern while Terwisga [26] adds that a pre-duct reduces the flow separation at the aft resulting in a reduction of viscous pressure resistance.

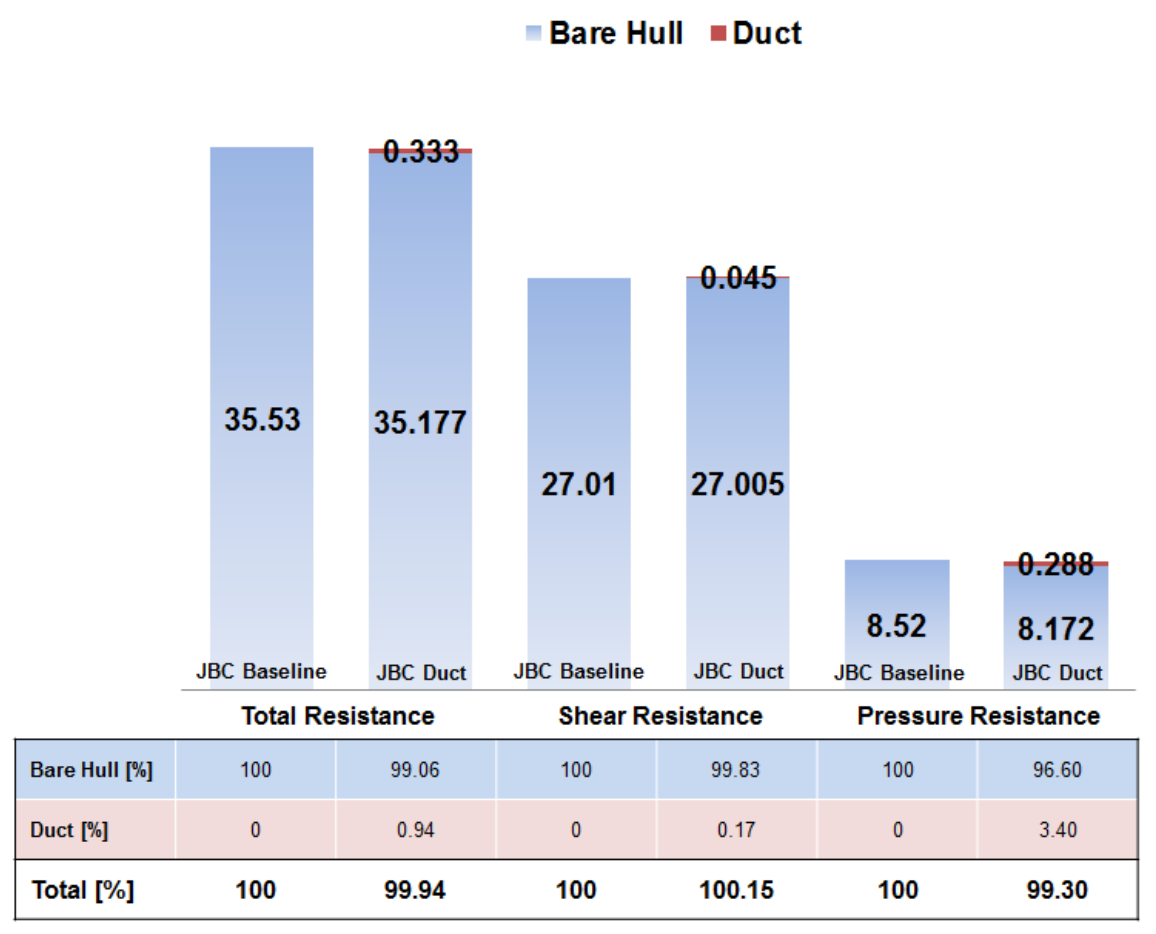

Figure 14 - Resistance force breakdown for both designs operating in design condition

As exhibited in Figure 14, the simulation results indicate that the ducted hull resulted in a very small total resistance reduction of $0.06 \%$. Although the magnitude of reduction lay within the simulation uncertainty the components contributing to the total resistance were further analysed. The resistance breakdown demonstrated that the ESD reduced the resistance of the bare hull by around $1 \%$. More specifically, this beneficial decrease was determined due to a noticeable decrease in the bare hull 
pressure resistance of $3.4 \%$. This would indicate a reduction in flow separation and less energy lost to the generated vortices. This was further justified by comparing the Wall Shear Stress values between the geometries as presented in Figure 15 with values higher than 0 indicating regions of flow separation and reversed flow. Although a larger separation area appeared on the JBC hull with duct, the magnitude of separation was higher on the JBC without duct as indicated by the contour plot.

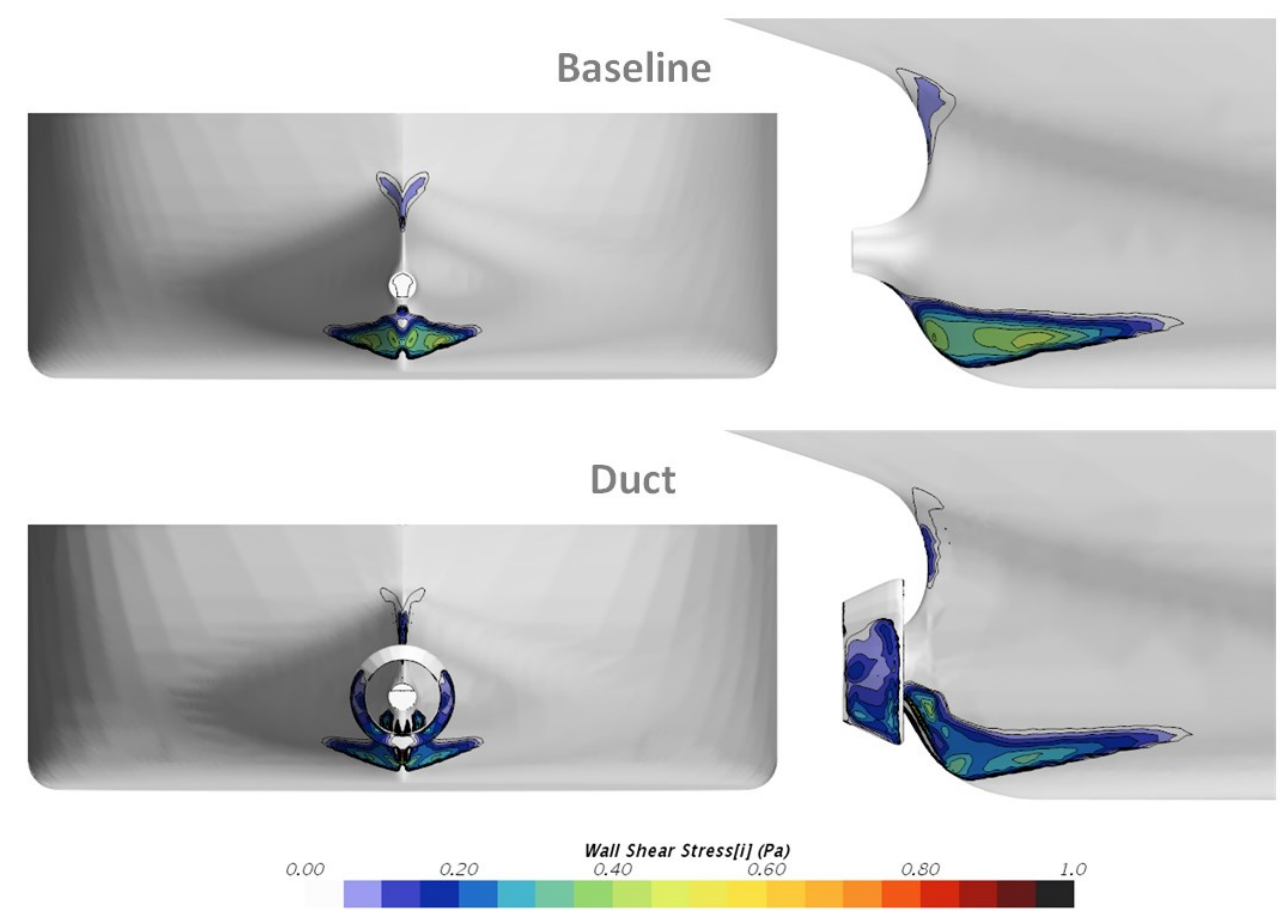

Figure 15 - Flow Separation Comparison (1)

It is evident that the baseline hull without duct experiences stronger separation of flow. This was further confirmed when analysing the wake at different planes upstream of the duct highlighting reduced extents of separation around the ship hull (Figure 16).

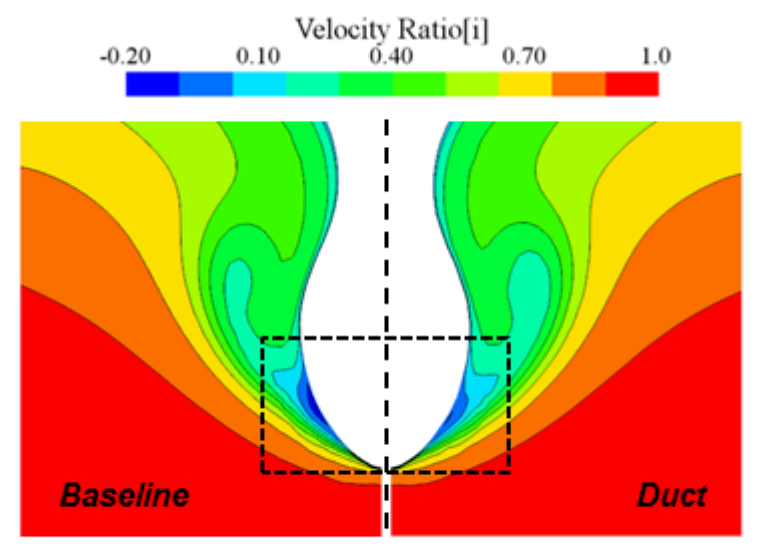

Plane Position: 0.0375 Lpp

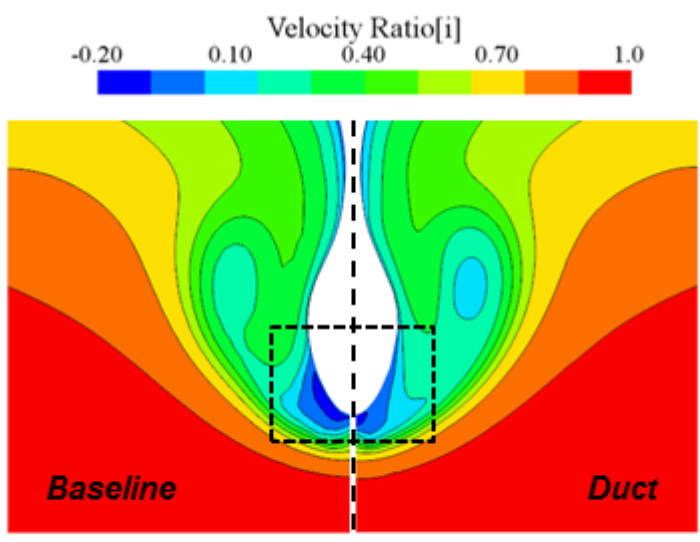

Plane Position: $0.0286 \mathrm{Lpp}$

Figure 16 - Flow Separation Comparison (2) 
When analysing the limiting streamlines at the stern of the bulk carrier in the absence of the duct, an area of recirculation just below the bossing (Figure 17 LHS) was present. These effects are reduced once the duct is installed (Figure $17 \mathrm{RHS}$ ). For the purpose of clarity only the outline of the duct is included in the respective figure.
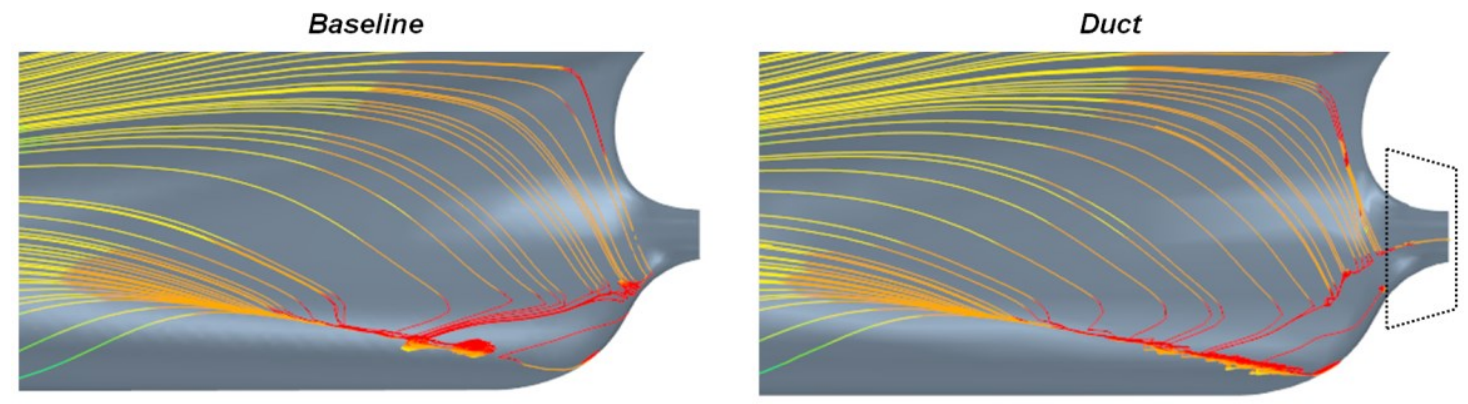

Figure 17 - Limiting Streamlines for baseline design (LHS) and duct design (RHS) without duct visualisation

It is worth highlighting that the duct is producing only net drag in nominal conditions while the situation will change when the effect of propeller action is included. However, as previously indicated, the suction effect of the duct reduced the resistance of the hull. After investigating the effect of the installation of a duct on the stern flow of the JBS upstream of the duct, the WAT was used to identify changes downstream of the duct in the propeller plane.

\section{WAT - WAKE FIELD ANALYSIS}

Figure 18 shows an example of how to use the WAT in combination with the flow solver post processing abilities. Both the extracted contour plot of the wake disc and the wake probe points are located in the same plane. The extracted flow field data is analysed by the WAT that allows the quantification of the velocity field in useful measures, such as the average axial velocity ratio and the maximum velocity variation. For better understanding the velocity distribution graph (bottom plot) and the corresponding values in the wake field analysis (top right plot) for the radius $\mathrm{r} / \mathrm{R}=0.6$ are highlighted. Here, the presence of the duct causes a high fluctuation of the axial velocity along the radius $r / R=0.6$ including an area of reversed flow. This results in a high maximum velocity variation and a low average velocity ratio. Furthermore the maximum velocity distribution (purple curve) reveals that the area of reversed flow also influences the neighbouring radii, $r / R=0.5$ and $r / R=0.7$, which leads to an overall increased mean wake variation and mean wake fraction. 

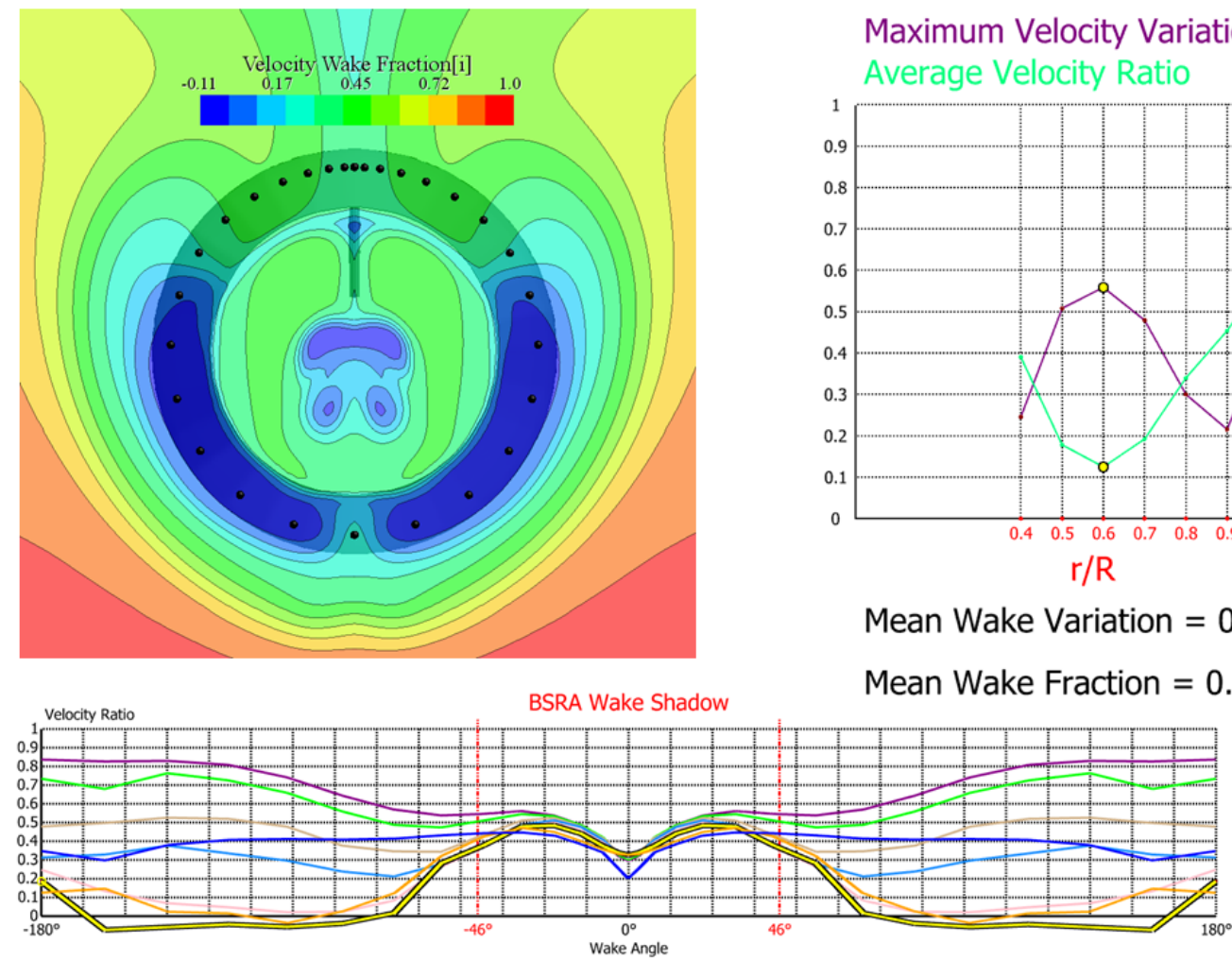

Maximum Velocity Variation

Average Velocity Ratio

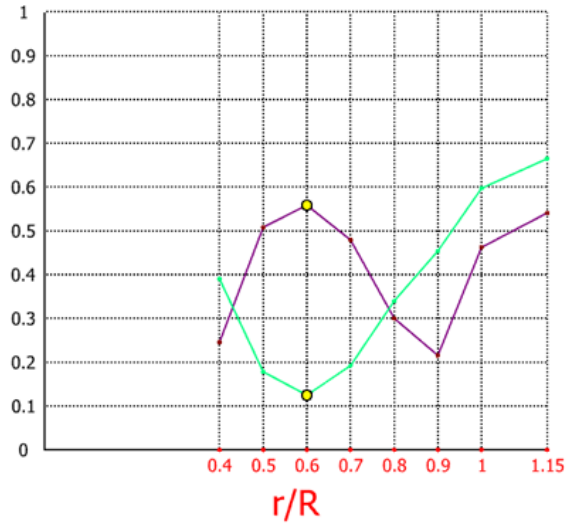

Mean Wake Variation $=0.422$

Mean Wake Fraction $=0.591$

Figure 18 - Wake Flow Analysis using a contour plot and the WAT for $r / R=0.6$

Table 8 shows an overview of the wake field contour plots along with the basic wake analysis of the WAT in the propeller plane for each computed variant. Each row in the table compares the different design variants for the same condition, i.e. same speed and draft. In the further analysis the various operating conditions were compared for each JBC design, with and without duct respectively, in order to understand the impact caused by the change in draft and ship velocity on the wake field quality. Both JBC design variants were then compared against each other for the same operating condition to understand how the introduction of the duct influences the velocity distribution of the nominal wake fields. 
Table 8 - Variation study results

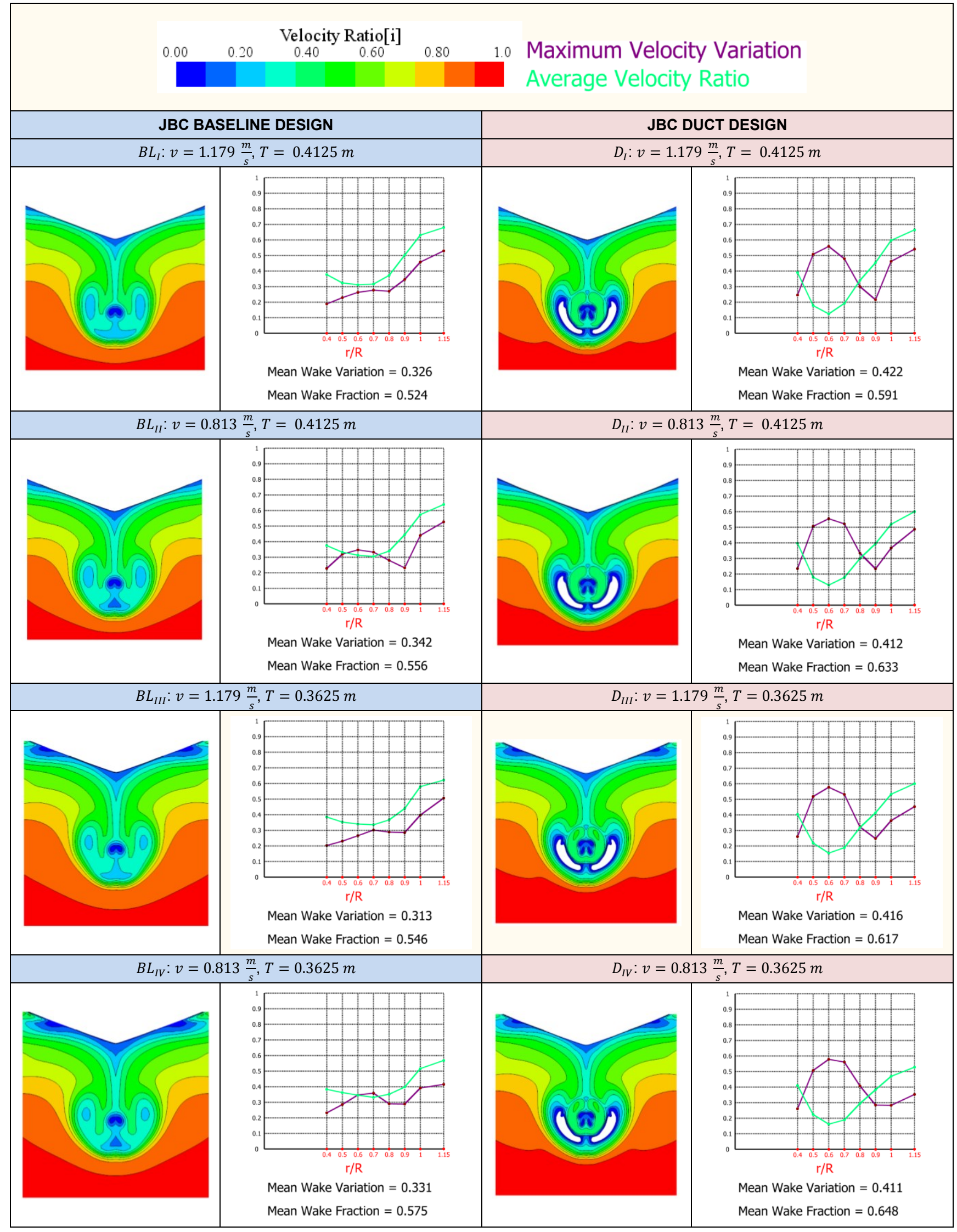


Firstly, the different operating conditions for the baseline design are compared with each other. It can be seen that the influence of the change of speed on the mean wake fraction is higher than the change of draft. In addition, a lower ship speed has a negative effect on the velocity variation which locally increases at the radius $\mathrm{r} / \mathrm{R}=0.6$ corresponding to the duct radius. Between the different conditions both the mean wake fraction and the wake variation varied by $10 \%$. Secondly, the assessment of the basic wake analysis for the Duct design shows a similar variation in mean wake fraction of about $10 \%$. However, the introduction of the duct further suppresses the wake variation to a range of around $3 \%$ between the variants. The presence of the duct causes the velocity variation over each radius to be less dependent on the operational condition. Once again, the ship speed shows a higher influence on the mean wake fraction as it directly depends on the magnitude of incoming flow. Thirdly, comparing the JBC design variants directly against each other (duct versus no duct) reveals that the introduction of the duct increased the nominal mean wake fraction by around $13 \%$ in all operational conditions and the mean wake variation by up to $32 \%$. A more inhomogeneous inflow is produced which might make the propeller susceptible to cavitation.

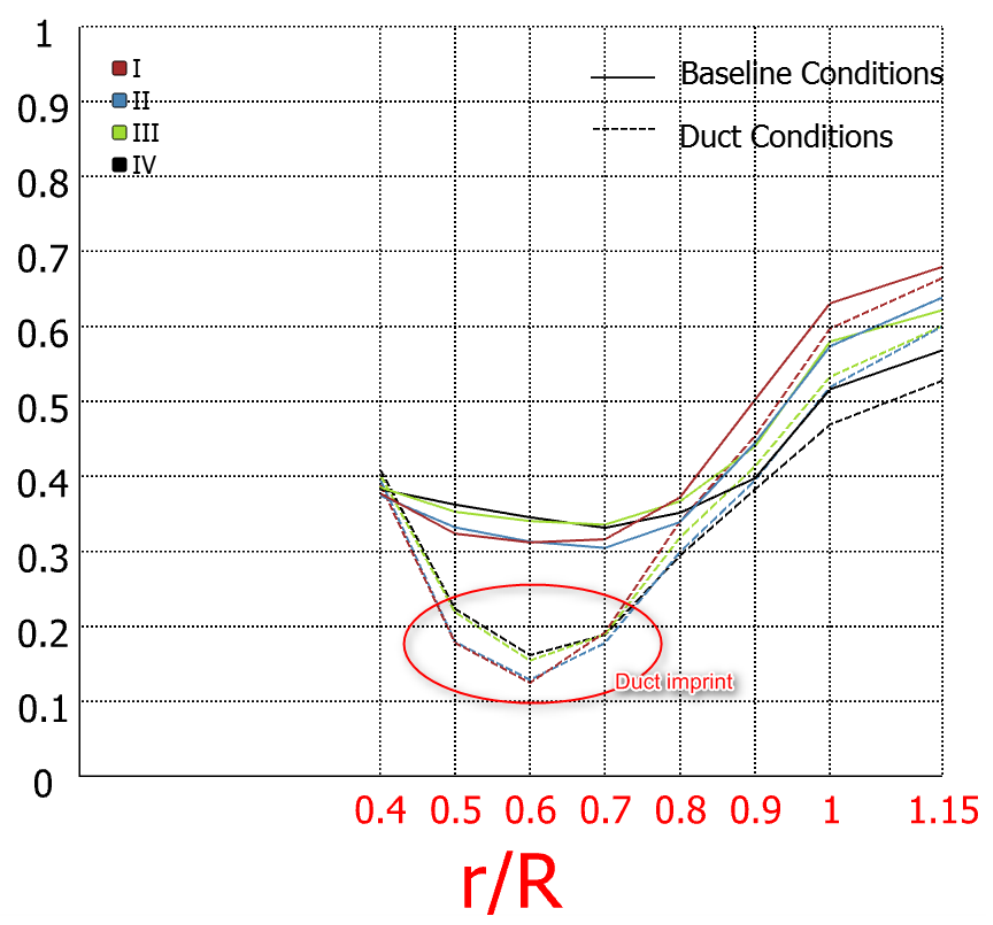

Figure 19 - Average Velocity Ratio for all calculated variants

The duct created a clearly visible imprint (see Figure 19) at the radius $r / R=0.6$ with locally stagnated/reversed flow. This behaviour can also be identified in the experimental results, though not as strong as calculated by the CFD solver which over predicts the reversed flow. One can also note an increase in maximum velocity variation at the same radii (see Figure 20). Interestingly, the presence of the duct decreased the velocity variation at the outer radii which could improve the ship's propulsive performance and reduce the risk of vibration and cavitation risk. With regards to the latter, B.S.R.A. criterion IV and $\mathrm{V}$ will provide further details. 


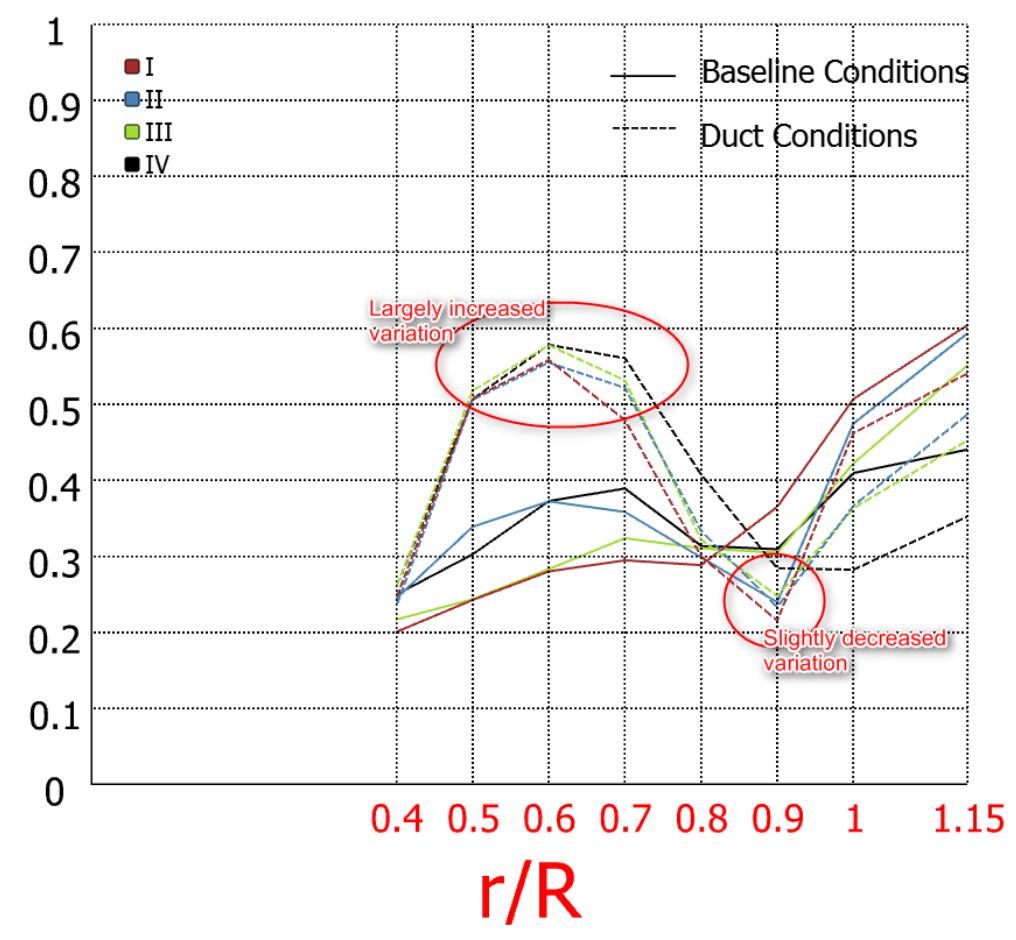

Figure 20 - Maximum Velocity Variation for all calculated variants

The above analysis showed that the nominal wake field quality reduced with the installation of an energy saving duct. However, the duct as an energy saving device is expected to improve the propulsion characteristics of the hull-propeller interaction system. Thus, the application of the WAT demonstrated that the performance of this type of ESD cannot be judged by analysing the nominal wake field. As can be seen in Figure 21 the flow field around the duct showed a significant area of separation. Once the propeller is taken into consideration the induced suction effect on the velocity flow field would change the angle of attack of the flow on the duct which may generate forward thrust. This would have a significant effect not only on the flow field in the propeller plane but also on the mode of operation of the duct itself. Hence, this Energy Saving Device is yet to be numerically investigated in self-propulsion conditions to fully understand its performance. Reference can be made to the experimental results of the JBC in self-propulsion conditions as well as to the full scale self-propulsion CFD study carried out by [27].

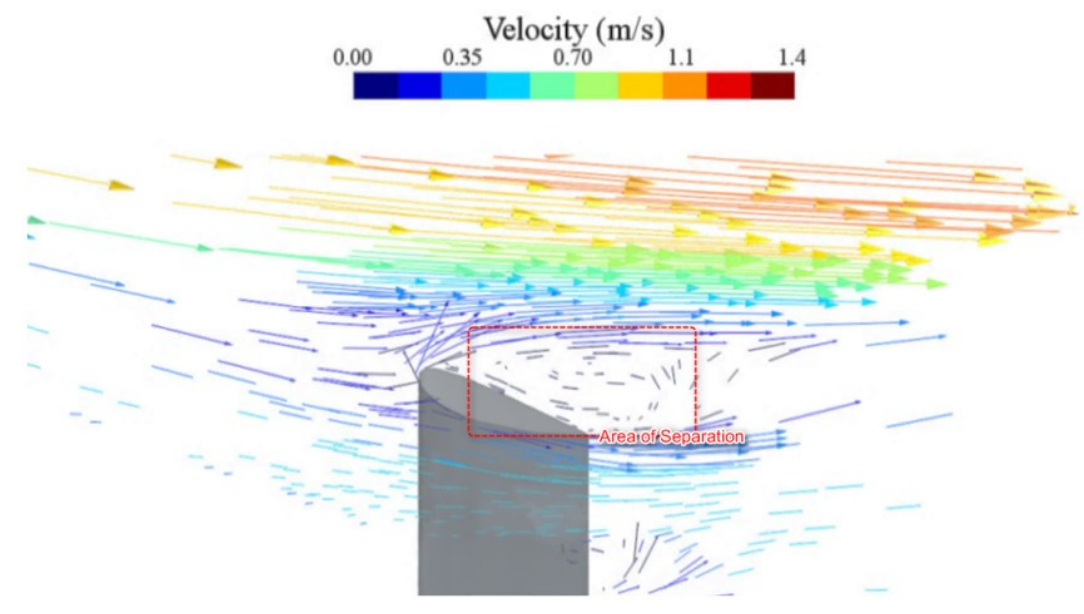

Figure 21 - Separation around duct at maximum width of the duct 
Finally, the assessment of the custom extension of the WAT predicts whether or not the wake fields fulfil the B.S.R.A. criteria. For the baseline design all measured radii were considered. For the duct design, however, the authors suggest to exclude those radii that are located within the duct making the B.S.R.A. criteria more suitable for ducted hull designs. Since the fractional radius $\frac{r}{R}=0.7$ is included in the B.S.R.A. criterion II this was chosen to be the minimum radius for the further analysis. For the sake of completeness an analysis over all calculated radii was performed as well.

In Table 9, a fulfilled criterion is indicated by green colour whereas a violated criterion is coloured red. For the B.S.R.A. criteria I and II both sets of radii were analysed. The thresholds for each criterion are given in the table as well.

Table 9 - Results of B.S.R.A. criteria for (green $=$ fulfilled, red $=$ not fulfilled)

\begin{tabular}{|c|c|c|c|c|c|c|c|}
\hline \multirow{2}{*}{ Criteria } & \multicolumn{2}{|c|}{ B.S.R.A. I } & \multicolumn{2}{|c|}{ B.S.R.A. II } & B.S.R.A. III & B.S.R.A. IV & B.S.R.A. V \\
\cline { 2 - 8 } & $w_{\max , \varphi_{B}}<0.75$ & $w_{\text {max,disc }}<1.7 \bar{w}_{0.7}$ & $\varphi_{B}>92.5^{\circ}$ & $\left(\sigma_{n I}\right)_{1.0}>x$ & $\frac{1}{r / R}\left|\frac{d w / d \varphi}{(1-w)}\right|<1.0$ \\
\hline Radii $\frac{\boldsymbol{r}}{\boldsymbol{R}}$ & $\mathbf{0 . 4 - 1 . 1 5}$ & $\mathbf{0 . 7 - 1 . 1 5}$ & $\mathbf{0 . 4 - 1 . 1 5}$ & $\mathbf{0 . 7 - 1 . 1 5}$ & $\mathbf{1 . 0}$ & $\mathbf{1 . 0}$ & $\mathbf{0 . 7 - 1 . 1 5}$ \\
\hline $\boldsymbol{B} \boldsymbol{L}_{\boldsymbol{I}}$ & 0.718 & & 0.789 & & $44.6^{\circ}$ & 0.334 & 3.27 at 0.9 \\
$\boldsymbol{B} \boldsymbol{L}_{\boldsymbol{I I}}$ & 0.749 & & 0.865 & & $43.0^{\circ}$ & - & 3.34 at 0.9 \\
$\boldsymbol{B} \boldsymbol{L}_{\boldsymbol{I I I}}$ & 0.760 & & 0.795 & & $43.1^{\circ}$ & - & 3.40 at 0.9 \\
$\boldsymbol{B} \boldsymbol{L}_{\boldsymbol{I V}}$ & 0.803 & & 0.856 & & $42.5^{\circ}$ & - & 3.50 at 0.9 \\
$\boldsymbol{D}_{\boldsymbol{I}}$ & 0.796 & 0.702 & 1.07 & 0.979 & $51.1^{\circ}$ & 0.345 & 2.79 at 0.8 \\
$\boldsymbol{D}_{\boldsymbol{I I}}$ & 0.786 & 0.713 & 1.07 & 1.03 & $41.1^{\circ}$ & - & 2.82 at 0.8 \\
$\boldsymbol{D}_{\boldsymbol{I I I}}$ & 0.802 & 0.722 & 1.08 & 1.03 & $42.4^{\circ}$ & - & 2.88 at 0.8 \\
$\boldsymbol{D}_{\boldsymbol{I V}}$ & 0.798 & 0.734 & 1.08 & 1.05 & $33.6^{\circ}$ & - & 2.95 at 0.8 \\
\hline
\end{tabular}

B.S.R.A. Criterion I - Two baseline design conditions, $B L_{I}$ and $B L_{I I}$, fulfilled the first criterion. Baseline conditions $B L_{I I I}$ and $B L_{I V}$ (low draft) violated the criterion. Figure 22 compares the wake along the wake angle at the fractional radius $\frac{r}{R}=0.4$ within the wake shadow area as these wake profiles are the reason for the criteria violation. Due to the steep increase of the wake for all profiles except $B L_{I}$ and $B L_{I I}$ the threshold $w_{\max }<0.75$ is exceeded. When excluding the fractional radii $\frac{r}{R}=0.4-0.6$ all calculated conditions for the ducted hull fulfil the criteria.

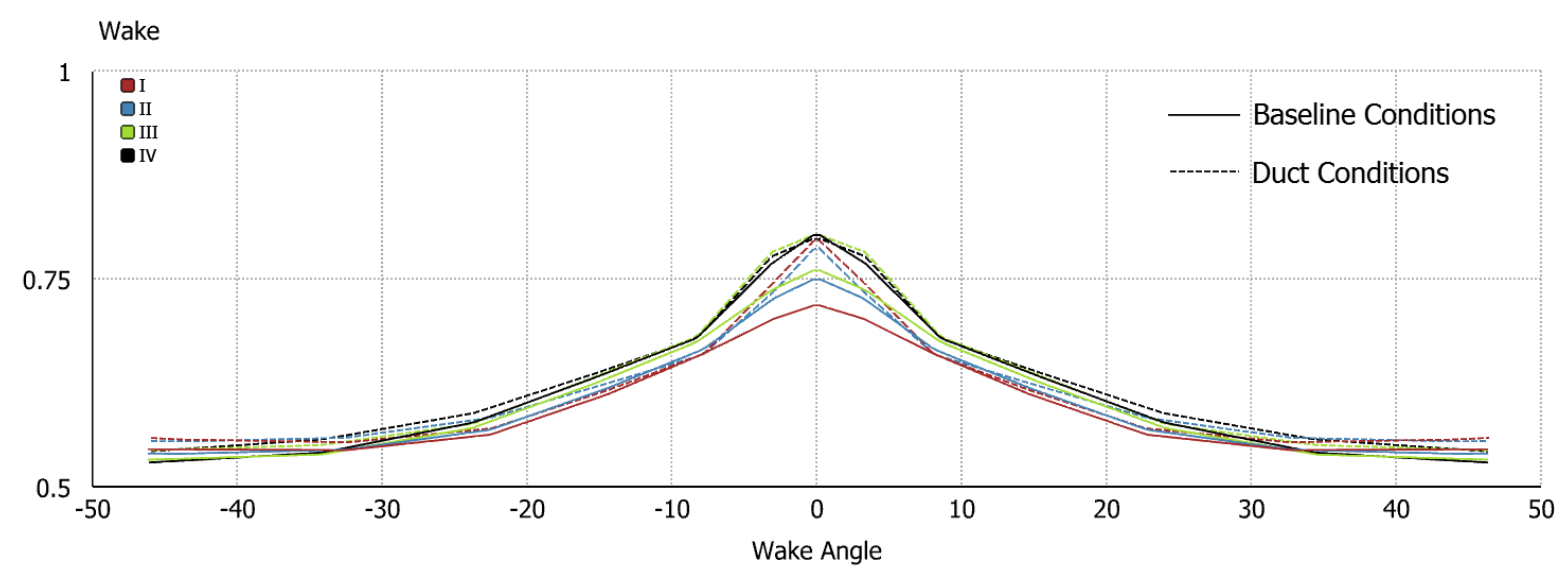

Figure 22 - Comparison of the wake distributions at the radius $\frac{r}{R}=0.4$ within the wake shadow for all conditions 
B.S.R.A. Criterion II - The second criterion was fulfilled by all simulated conditions for both the baseline design and the duct design. Even though the maximum wake on the entire disc including areas with stagnated/reversed flow was considered, the criteria seems to be weak for wake fields with an already high average wake on the effective fractional radius $\frac{r}{R}=0.7$. The velocity distribution for the radius $\frac{r}{R}=$ 0.7 still shows a clear imprint of the duct, thus the average wake was quite high.

B.S.R.A. Criterion III - The third criterion, an acceptable wake peak width with respect to the wake shadow width, was violated for all conditions $\varphi_{B}<\left(\varphi_{\max }\right)_{1.0}$. This could be expected from a high block coefficient $\left(c_{B}\right)$ ship such as the JBC. When comparing both designs at operational design conditions, an improvement was found for the duct retrofitted vessel with an increase of $\Delta \varphi_{B}=17.5^{\circ}$. This supports the above statement that indicates the duct diminishes the wake variation at the outer radii. Each computed wake field featured a single wake peak with a width range of $33.6^{\circ} \leq \varphi_{B} \geq 51.1^{\circ}$ and a target of $\left(\varphi_{\max }\right)_{1.0}=92.5^{\circ}$. Consequently, each wake field showed a large gradient within the wake shadow area. This could lead to a rapid collapse of cavitation close to the propeller and the hull causing damage and noise. However, before coming to any conclusions, the fourth B.S.R.A. criterion has to be evaluated to assess the risk of cavitation.

B.S.R.A. Criterion IV - In order to evaluate the fourth criterion, estimations required the propeller rotation rate (rps) values. Since no self-propulsion simulations were performed, self-propulsion details were derived from the experimental data. Since the change of required rps for all other conditions was unknown the change of the tip cavitation number could not be calculated with accuracy. Although the change in static pressure at the blade tip could be calculated by taking into account the changing draft, the dynamic pressure was unknown due to the change in rps. However, all additional conditions were defined by either a lower draft and/or a lower speed, thus the rps would drop due to the lower hull resistance. For the sake of simplicity the rps was kept constant corresponding to the design operating conditions for the hull with and without duct respectively. As shown in Figure 23 all data sets cover an area above the dividing band (red area). Consequently, it can be concluded that all calculated variants fulfilled the fourth B.S.R.A. criterion.

Tip Cavitation Number

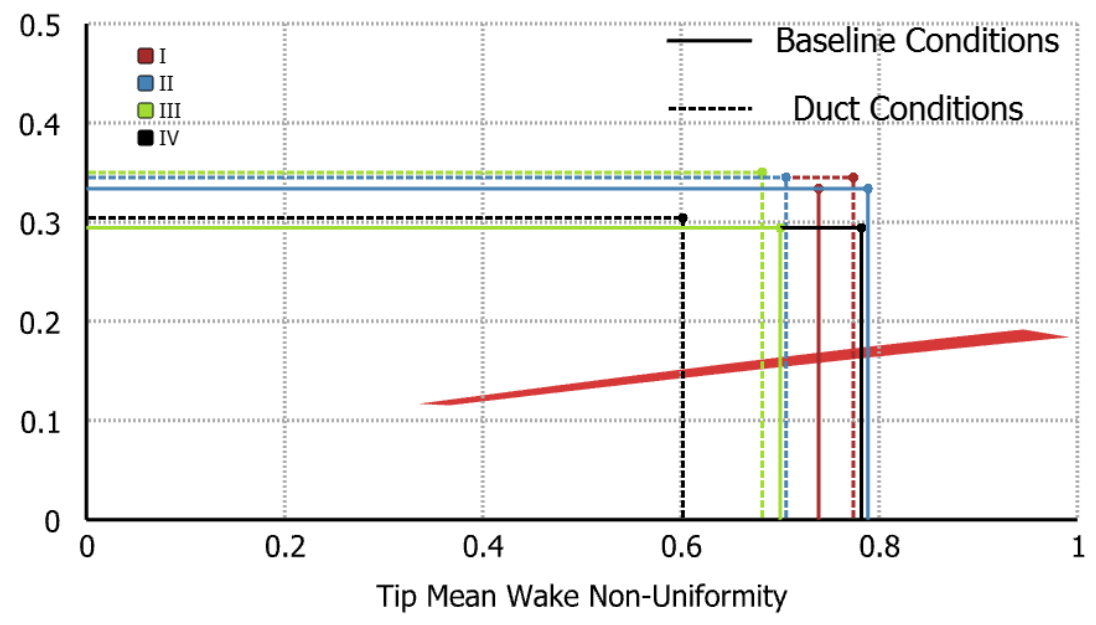

Figure 23 - Graphical WAT output of B.S.R.A. criterion IV for baseline and duct design in design conditions

Since there is no high risk of cavitation, as indicated by B.S.R.A. criterion IV, the high wake gradient in the wake shadow area as indicated by B.S.R.A. criterion III will have a minor impact on the ship performance. If, however, high velocity gradients also develop for higher ship speeds which can be expected, cavitation becomes more likely with an immediate collapse of cavitation volume that will 
create high pressure fluctuation near the propeller and on the hull, thus decreasing the ship performance.

B.S.R.A. Criterion V - Although the B.S.R.A. criterion IV was fulfilled, the B.S.R.A. criterion $V$ was still evaluated to check for high wake gradients. None of the simulated conditions fulfilled the fifth criterion. Table 9 presents the highest local wake gradient within the wake field as well as the radius where this value was found. The local wake gradients per unit axial wake velocity are very high compared to the criteria threshold of one, indicating that if the propeller would be susceptible to cavitation there would be high instantaneous variations of cavitation volume producing impulsive pressures on the hull. This might become a problem for higher ship speeds as well.

When comparing the B.S.R.A. criteria evaluation for both design conditions $B L_{I}$ and $D_{I}$, it can be concluded that B.S.R.A analysis predicts a slightly worse performance for the ducted hull in terms of vibration impact on the stern hull. For all criteria the combination of a low speed and a low draft produced a critical flow field environment. 


\section{Conclusion}

The present study introduced the functionality of a self-developed wake field analysis tool (WAT) and successfully applied the tool to a set of numerical propeller wake fields calculated for the Japan Bulk Carrier (JBC) test case.

While it has become a standard in industry to carry out both experimental towing tank tests and numerical towing tank simulations to design a ship and its appendages, the detailed analysis of a wake field is generally not performed. By analysing and comparing each wake field using well-established wake quality criteria, it was shown that the WAT can be used to quickly classify propeller wake fields in a preliminary design stage. This makes the WAT a powerful tool for Naval Architects to overcome timeconsuming CFD pre- and post-processing procedures. By implementing the tool into a software chain by employing to commercial software tools, it was further shown that the WAT wake analysis can be fully automated thus allowing to run variation and optimisation studies.

After performing a verification and validation study for the numerical towing tank setup to identify numerical errors and uncertainties, the WAT analysis was applied to eight different nominal wake fields, resulting from the simulation of both JBC design variants (hull with and without duct) at four different operating conditions (see Table 1). The WAT was also able to identify small changes in the nominal propeller wake flow, even those that could have not been easily identified visually. The basic analysis of the baseline design conditions (hull without duct) showed that the mean wake fraction and the mean wake variation varied by around $10 \%$, indicating that the slight changes of the operating conditions do not have a major impact on the ship performance. The wake fields of the JBC with duct showed that the mean wake variation (varied by $3 \%$ ) became less dependent of the operational conditions due to the presence of the ESD upstream of the propeller. The comparison of both JBC hull designs for the same operational condition showed that the wake field quality decreased due to stagnated/reversed flow on the inner radii caused by the presence of the duct. However, the local wake variation decreased at the outer radii indicating a more uniform inflow. Due to the overall decrease of the nominal wake field quality (judged by the mean wake fraction and the mean wake variation) between both JBC design variants, it seems to be unreasonable to use such measures to predict the self-propulsion performance improvement of a hull appended with a duct. Here it seems necessary to directly perform self-propulsion tests/simulations as already stated by Visonneau et al [27].

The evaluation of vibration levels on the hull surface and the estimation of risk of cavitation were performed by applying the B.S.R.A. criteria to the numerically measured wake fields. Not all B.S.R.A. criteria were fulfilled. This indicates that certain levels of vibration can be expected. However, the results also showed that the propeller is not prone to adverse cavitation dynamics for the calculated operational conditions. Furthermore, the WAT B.S.R.A. analysis showed that by lowering the draft from $\mathrm{T}_{\mathrm{A}}=16.5 \mathrm{~m}$ to $\mathrm{T}_{\mathrm{A}}=14.5 \mathrm{~m}$ and the speed from $\mathrm{v}=14.5 \mathrm{kn}$ to $\mathrm{v}=10 \mathrm{kn}$ (full scale) the working conditions for the propeller became critical. Since this operational condition represented the most extreme case, it can be concluded that the JBC hull was not designed to operate far away from its design point.

Further investigation (i.e. experimental and numerical testing) would be necessary to clarify how the B.S.R.A. criteria could be modified to accurately predict the performance of a ducted hull. Although the BSRA criteria were based on open propeller operations, the wake field altered by the presence of an ESD could still suit the method if the flow remains nominal in character. In the present case, the nominal flow altered by an upstream located pre-duct would be different with the propeller operating, so any cavitation activity might be expected to be better than the nominal analysis would indicate. Alternatively, an actuator disk could be used to mimic the flow acceleration. In addition, it should be studied whether the criteria are of equal importance or if a weighting factor should be attached to each criterion. It should also be mentioned that the WAT can be applied to effective numerical wake fields as well as experimental digitally measured wake fields. Therefore, it can also serve as useful tool in validating numerical wake fields that can be directly compared to the experimental data. An ongoing development of the WAT would be desirable to make the code suitable to read flow field data provided in Cartesian 
coordinates. With the tool being editable in a programming environment, the basic structure of the code could be adapted to other problems concerning the quality of stern flow fields, such as the hull pressure analysis proposed by Holden [28].

\section{Acknowledgement}

Results were obtained using the EPSRC funded ARCHIE-WeSt High Performance Computer (www.archie-west.ac.uk). EPSRC grant no. EP/K000586/1.

\section{References}

1. Carlton, J.S., Marine Propellers and Propulsion - The Ship Wake Field. Third Edition ed. 2012, Butterworth-Heinemann: Elsevier Ltd.

2. SNUTT. Seoul National University Towing Tank - Facility. 2017 [cited 2017; Available from: snutt.snu.ac.kr/?page_id=110.

3. Atlar, M., D. Wang, and E.J. Glover, Experimental investigation into the impact of slipstream wash of a podded propulsor on the marine environment. Journal of the Engineering for Maritime Environment, 2007. Proc. IMechE (Vol. 221 Part M).

4. Aktas, B., et al., Propeller cavitation noise investigations of a research vessel using medium size cavitation tunnel tests and full-scale trials. Ocean Engineering, 2016(120): p. 122-355.

5. Carlton, J.S. The Propulsion of Large Container Ship, A Note on the Propulsion Options. in Proceedings Symposium on Mega-Container Ship in Future, the Kansai Society of Naval Architects. 2001. Kobe, Japan.

6. Huse, E., EFFECT OF AFTERBODY FORMS AND AFTERBODY FINS ON THE WAKE DISTRIBTUTION OF SINGLE-SCREW SHIPS. 1974.

7. Odabasi, A.Y. and P.Fitzsimmons, Alternative methods for wake quality assessment. International Shipbuilding Progress, 1978. 25(282).

8. Larsson, L., F. Stern, and V. Bertram, Benchmarking of Computational Fluid Dynamics. Journal of Ship Research, 2003. vol. 47(No. 1): p. pp 63-81.

9. Visonneau, M. A step towards the numerical simulation of viscous flows around ships at full scale - Recent achievements within the European Union Project EFFORT. in Royal Institute of Naval Architecture Marine CFD. 2005. Southampton, France.

10. Hanninen, S. and T. Mikkola. Computation of ship-hull flows at model-and full-scale Reynolds numbers. in Numerical Ship Hydrodynamics Seminar, Maritime Institute of Finland, Turku. 2006. 
11. Wang, J., et al., Numerical simulation of viscous wake field and resistance prediction around slow-full ships. Chinese Journal of Hydrodynamics, 2010. 25(5): p. 648-654.

12. Mizzi, K., et al., ISSUES WITH ENERGY SAVING DEVICES AND THE WAY FORWARD in SCC Conference 2015. 2015, University of Strathclyde: Glasgow.

13. Ok, J.-P., Numerical investigation of scale effects of Schneekluth's duct. 2004: Arbeitsbereiche Schiffbau der Techn. Univ.

14. Stein, N.v.d., 12 Jahre Schneekluth-Zuströmdüse. HANSA, 1996. no. 7.

15. Han, J., et al. 'Application of Flow Control Devices to Improve Propeller Cavitation Performances'. in 9th Symposium on Practical Design on Ships and Other Floating Structures, PRADS2004. 2006. Lübeck-Travemünde.

16. Johannsen, C. Recent considerations on dealing with propeller induced hull pressure pulses. in International Conference on ship and shipping research, NAV2000. 2000. Venice.

17. Maasch, M., et al., Calm water resistance and self propulsion simulations including cavitation for an LNG carrier in extreme trim conditions, in Shipping in Changing Climates SCC 2017. 2017: London.

18. (NMRI), N.M.R.I., Y.N. University, and S.B.R.C.o.J. (SRC). JAPAN Bulk Carrier (JBC). 2015 [cited 2017; Available from: http://www.t2015.nmri.go.jp/jbc.html.

19. T. Xing, F.S., Factors of Safety for Richardson Extrapolation. Journal of Fluids Engineering, 2010. 132: p. 061403- 1 - 13.

20. NMRI. A Workshop on CFD in Ship Hydrodynamics. 2015.

21. Ferziger, J.H. and M. Peric, Computational methods for fluid dynamics. 2002: Springer.

22. Date, J.C. and S.R. Turnock, A study into the techniques needed to accurately predict skin friction using RANS solvers with validation against Froude's historical flat plate experimental data. (Ship Science Reports, (114)) ed. 1999, Southampton, UK: University of Southampton, Department of Ship Science. 62pp.

23. Richardson, L.F., The approximate arithmetical solution by finite differences of physical problems involving differential equations, with an application to the stresses in a masonry dam. Transactions of the Royal Society of London, 1910. 210: p. 307-357.

24. Roache, P.J., Verification and Validation in Computational Science and Engineering. 1998: Hermosa. 
25. Celik, I.B., Ghia, U., Roache, P. J., Freitas, C. J., Coleman, H. \& Raad, P. E., Procedure for Estimation and Reporting of Uncertainty Due to Discretization in CFD Applications. Journal of Fluids Engineering, 2008: p. 130.

26. Terwisga, T.V., On the working principles of Energy Saving Devices., in 3rd International Symposium on Marine Propulsors. 2013: Tasmania, Australia.

27. Visonneau, M., et al., Local and Global Assessment of the Flow around the Japan Bulk Carrier with and without Energy Saving Devices at Model and Full Scale, in 31st Symposium on Naval Hydrodynamics 2016: Monterey, California.

28. Carlton, J.S., Marine Propellers and Propulsion - Propeller, Ship and Rudder Interaction. Third Edition ed. 2012, Butterworth-Heinemann: Elsevier Ltd. 